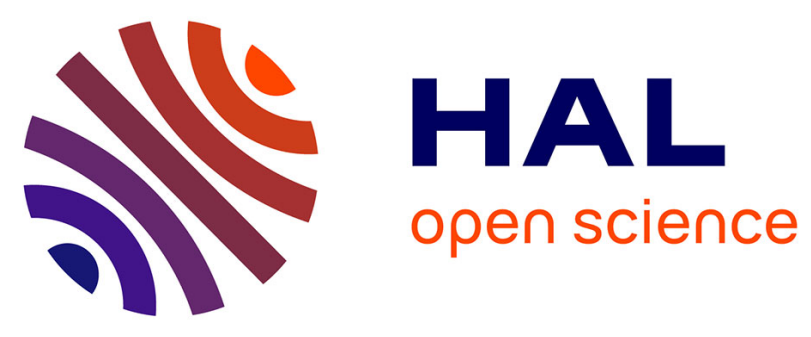

\title{
Pseudopilin residue E5 is essential for recruitment by the type 2 secretion system assembly platform.
}

\author{
Mangayarkarasi Nivaskumar, Javier Santos-Moreno, Christian Malosse, \\ Nathalie Nadeau, Julia Chamot-Rooke, Guy Tran van Nhieu, Olivera \\ Francetic
}

\section{To cite this version:}

Mangayarkarasi Nivaskumar, Javier Santos-Moreno, Christian Malosse, Nathalie Nadeau, Julia Chamot-Rooke, et al.. Pseudopilin residue E5 is essential for recruitment by the type 2 secretion system assembly platform.. Molecular Microbiology, 2016, 101 (6), pp.924-941. 10.1111/mmi.13432 . pasteur-02282206

\section{HAL Id: pasteur-02282206 \\ https://hal-pasteur.archives-ouvertes.fr/pasteur-02282206}

Submitted on 9 Sep 2019

HAL is a multi-disciplinary open access archive for the deposit and dissemination of scientific research documents, whether they are published or not. The documents may come from teaching and research institutions in France or abroad, or from public or private research centers.
L'archive ouverte pluridisciplinaire HAL, est destinée au dépôt et à la diffusion de documents scientifiques de niveau recherche, publiés ou non, émanant des établissements d'enseignement et de recherche français ou étrangers, des laboratoires publics ou privés. 
1 Pseudopilin residue E5 is essential for recruitment by the type 2 secretion system assembly

2 platform

3

4 Mangayarkarasi Nivaskumar ${ }^{1,2 \#_{\star}}$, Javier Santos-Moreno ${ }^{2-6, *}$, Christian Malosse $^{7}$, Nathalie

5 Nadeau $^{1}$, Julia Chamot-Rooke ${ }^{7}$, Guy Tran Van Nhieu ${ }^{3-6}$ and Olivera Francetic ${ }^{1,8}$

6

$7 \quad{ }^{1}$ Laboratory of Macromolecular Systems and Signalling, Institut Pasteur, Department of

8 Microbiology, CNRS ERL3526, 25 rue du Dr Roux, 75724 Paris, Cedex 15, France

9 2Université Paris Diderot (Paris 7) Sorbonne Paris Cité

$10{ }^{3}$ Laboratory of Intercellular Communication and Microbial Infections, CIRB, Collège de

11 France, Paris France

$12{ }^{4}$ Institut National de la Santé et de la Recherche Médicale (Inserm) U1050, France

$13{ }^{5}$ Centre National de la Recherche Scientifique (CNRS), UMR7241, France

$14{ }^{6}$ MEMOLIFE Laboratory of Excellence and Paris Science Lettre, France

$15{ }^{7}$ Structural Mass spectrometry and Proteomics unit, CNRS UMR3528, Institut Pasteur, Paris,

16 France

17 \#Present address: Department of Chemistry, National University of Singapore, 3 Science

18 Drive, Singapore 117543

$19{ }^{*}$ Co-first authors

$20 \quad{ }^{8}$ Correspondence: ofrancet@pasteur.fr

$21 \quad$ Tel: 33140613681

22 Fax: +33145688960

23 Running title: Pseudopilin interactions with assembly factors

24 Key words: type 2 secretion system, type 4 pili, pilus assembly, bacterial two-hybrid,

25 assembly platform, protein interactions

26 


\section{Summary}

30 Type II secretion systems (T2SSs) promote secretion of folded proteins playing important 31 roles in nutrient acquisition, adaptation and virulence of Gram-negative bacteria. Protein

32 secretion is associated with the assembly of type 4 pilus (T4P)-like fibres called pseudopili. 33 Initially membrane embedded, pseudopilin and T4 pilin subunits share conserved 34 transmembrane segments containing an invariant Glu residue at the 5th position, E5. 35 Mutations of E5 in major T4 pilins and in PulG, the major pseudopilin of the Klebsiella T2SS 36 abolish fibre assembly and function. Among the four minor pseudopilins, only PulH required 37 E5 for secretion of pullulanase, the substrate of the Pul T2SS. Mass-spectrometry analysis of 38 pili resulting from the co-assembly of $\mathrm{PuIG}^{\mathrm{E5A}}$ variant and $\mathrm{PuIG}^{\mathrm{WT}}$ ruled out an $\mathrm{E} 5$ role in pilin 39 processing and $\mathrm{N}$-methylation. A bacterial two-hybrid analysis revealed interactions of the 40 full-length pseudopilins PulG and PulH with the PulJ-Pull-PulK priming complex and with the 41 assembly factors PulM and PuIF. Remarkably, PulG ${ }^{E 5 A}$ and $P u l H^{E 5 A}$ variants were defective 42 in interaction with PulM, but not with PulF and co-purification experiments confirmed the E543 dependent interaction between native PulM and PulG. These results reveal the role of E5 in 44 a recruitment step critical for assembly of the functional T2SS, likely relevant to T4P 45 assembly systems. 
51 Bacterial interactions with their environment involve the transport of proteins and other macromolecules across their cell envelope. In Gram-negative bacteria, several sophisticated

53 molecular machines facilitate protein secretion across two bilayer membranes (Costa et al., 54 2015). Among these, the type II secretion system (T2SS) promotes specific transport of 55 folded proteins across the outer membrane once they have reached the periplasmic compartment though the Sec or the Tat systems (Voulhoux et al., 2001; Korotkov et al., 2012; Nivaskumar and Francetic, 2014). T2SSs are structurally and functionally similar to type IV pilus (T4P) assembly systems (Berry and Pelicic, 2014). Unlike type IV pili, which extend beyond the bacterial surface to promote attachment and motility, the T2SS pseudopilus fibres are thought to be restricted to the periplasm. However, plate-cultured bacteria that overexpress T2SS-encoding genes present extended pseudopili (hereafter referred to as T2SS pili) on the cell surface, a feature that has facilitated their biochemical and structural characterisation (Sauvonnet et al., 2000; Durand et al., 2003; Kohler et al., 2004). T2SS pili are helical homo-polymers composed of one major pseudopilin subunit called PulG in K. oxytoca (Sauvonnet et al., 2000; Kohler et al., 2004). Mechanistic insights gained from their structure function analysis have revealed that interactions between neighbouring pilus subunits $\mathrm{P}$ and $\mathrm{P}^{+1}$ tightly correlate with fibre assembly and function in protein secretion (Campos et al., 2010). The fact that these essential contacts take place in the membrane implies a one-start helix rotational assembly mode for these and other type 4 pilus-like fibres (Nivaskumar et al., 2014).

Pseudopilus biogenesis is a multistep process that begins with co-translational insertion of pseudopilins in the inner membrane (IM) via the SRP and the Sec machinery

73 (Arts et al., 2007; Francetic et al., 2007). Pseudopilins are made as precursors anchored in 74 the IM through a positively charged $\mathrm{N}$-terminal peptide that is removed prior to assembly by 75 an inner membrane protease called the prepilin peptidase (Nunn and Lory, 1991). Following 
cleavage of this peptide after a conserved Gly residue at the base of the transmembrane segment, the $\mathrm{N}$-terminal domain of the prepilin peptidase transfers a methyl group to the new N-terminal residue of the mature pilin (Strom et al., 1993; Aly et al., 2013).

In addition to the highly abundant major pseudopilins that build the helical homopolymeric fibre, T2SSs contain four minor pseudopilins, essential for function. Purified globular domains of three of these minor subunits, Gspl, GspJ and GspK from enterotoxigenic Escherichia coli form a quasi-helical complex, which is predicted to cap the pseudopilus tip (Korotkov and Hol, 2008). Functional, biochemical and molecular dynamics analysis of their full-length equivalents in the Pul T2SS showed that Pull, PulJ and PulK selfassemble in the IM in the absence of other T2SS factors (Cisneros et al., 2012a). The PulJPull-PulK complex promotes initiation of pseudopilus assembly and presumably provides a template for subsequent incorporation of PulH and PulG subunits. Mutants lacking Pull, PulJ or PulK, but not PulH, assemble fewer PulG pili and are completely defective for secretion of the specific Pul T2SS substrate, pullulanase (PulA) (Cisneros et al., 2012a). Biochemical studies of the Pseudomonas aeruginosa Xcp T2SS show that the periplasmic domain of the PulH homologue $\mathrm{XcpU}$ forms a complex with other minor pseudopilins in vitro by binding to the PulJ homologue XcpW (Yanez et al., 2008; Korotkov and Hol, 2008; Douzi et al., 2009). In addition, the major pseudopilin XpsG of Xanthomonas campestris T2SS and the minor subunit $\mathrm{XpsH}$, a PulH homologue, interact directly in vivo (Hu et al., 2002). Based on these studies, PulH homologues are thought to provide a link between the priming complex and the major pseudopilins (Yanez et al., 2008; Korotkov and Hol, 2008; Douzi et al., 2009; Cisneros et al., 2012a).

Assembly of PulG fibres requires a functional complex called the assembly platform (AP) composed of the IM proteins PulC, PulF, PulL and PulM (Py et al., 2001; Possot et al., 2000). The cytoplasmic domain of PulL binds the hexameric ATPase PulE that provides energy for pseudopilus assembly (Abendroth et al., 2005; Camberg et al., 2007). Biochemical and structural analyses of AP components in T2SSs have provided molecular insights into their soluble domains and hints about their interactions and organization 
104 (Korotkov et al., 2011; Abendroth et al., 2004b; Abendroth et al., 2004a; Sandkvist et al., 105 1995; Johnson et al., 2007; Abendroth et al., 2005). This information has led to the current 106 T2SS model, where the IM platform promotes pseudopilus assembly within a cage-like compartment delimited by the secretin channel formed by a GspD dodecamer in the outer membrane and the GspD-interacting partner GspC in the IM (Gsp being a generic term to designate T2SS components) (McLaughlin et al., 2012).

The hydrophobic segments of major (pseudo)pilins in bacterial T4P and T2SSs are 111 highly conserved and rich in branched chain amino acid residues. In addition to the 112 conserved Gly residue preceding the prepilin peptidase cleavage site, mature subunits 113 contain an invariant Glu residue at position 5 (E5), which is located near the cytoplasmic side 114 of the transmembrane segment and is essential for function (Strom and Lory, 1991; Aas et al., 2007; Pugsley, 1993). In the T4P systems, residue E5 has been implicated in prepilin processing (Pasloske and Paranchych, 1988) or N-methylation (Strom and Lory, 1991; Aas 117 et al., 2007). Based on structural studies of T4P, it has also been proposed that E5 is 118 involved in docking of pilins to the growing fibre during assembly, by forming electrostatic contacts with the $\mathrm{N}$-terminal amine of the last pilin subunit incorporated in the pilus (Parge et al., 1995; Craig et al., 2006). In contrast, the E5V substitution in PulG from $K$. oxytoca T2SS did not affect 122 processing and N-methylation (Pugsley, 1993). Analyses of the PulG pilus structure and 123 assembly show that E5 is not implicated in docking, but participates in fibre stabilisation. 124 More precisely, once the protomer $\mathrm{P}$ has been extracted from the membrane, its E5 residue 125 interacts with two lysine residues (K28 and K35) of protomer $\mathrm{P}^{-3}$ (Campos et al., 2010; 126 Nivaskumar et al., 2014). However, the Ala substitutions of these Glu and Lys residues lead 127 to strikingly different phenotypes: while pulG ${ }^{K 28 A / K 35 A}$ mutants are fully functional in protein 128 secretion, pu/G ${ }^{E 5 A}$ mutants are completely defective. This and other data suggest an 129 additional role for E5 in a step preceding the docking step in the pilus assembly pathway 130 (Nivaskumar et al., 2014). With the exception of the interactions between EpsG and the AP 
131 component EpsL in Vibrio cholerae T2SS that have been revealed by chemical cross-linking

132 (Gray et al., 2011), little is known about the early events of the assembly process. To shed 133 some light on these steps, we sought to characterise interactions between minor and major 134 pseudopilins and to identify AP components that directly interact with pseudopilins using the 135 K. oxytoca T2SS as a model system. By combining bacterial two-hybrid and co-purification 136 approaches with mass spectrometry and functional analyses, we identified several 137 interacting partners of the K. oxytoca major pseudopilin PulG and revealed a novel role of the 138 conserved pseudopilin residue E5, which holds important clues to the assembly mechanism 139 of the T4P fibre family.

\section{Results}

As mentioned above, earlier studies showed that residue E5 is not required for PulG processing or N-methylation (Pugsley, 1993), while studies of T4P suggest the opposite (Strom and Lory, 1991; Aas et al., 2007). The assembly-defective pilin variants with E5 residue substitutions have been shown to co-assemble into pili in the presence of wild type pilins (Pasloske, et al., 1989; Aas et al., 2007). However, while N-terminal sequencing of Neisseria gonorrhoeae T4P made of PilE wild type and E5L variants revealed under-

151 methylation of the pilins, it was not clear whether this under-methylation was specific to 152 PilE ${ }^{E L L}$ (Aas et al., 2007). To clarify the role of E5 in PulG, we used a similar approach and 153 tested whether the assembly-defective $\mathrm{PuIG}^{\mathrm{E} \text { A }}$ variant can be co-assembled into pili with 154 PulG $^{\text {WT }}$. Therefore, we co-expressed the full set of genes encoding the Pul T2SS (on plasmid pCHAP8185, which includes PulG ${ }^{W T}$ ) with the gene encoding assembly-defective PulG ${ }^{E 5 A}$ variant (on plasmid pCHAP7790, Table 1). The surface proteins were isolated from these

157 bacteria and analysed by SDS-PAGE and Coomassie Blue staining (Experimental 158 Procedures and Figure 1A). A prominent band of approximately $14.5 \mathrm{kDa}$, the molecular 
159 mass of PulG, was present in the sheared fraction (Figure 1A, lane 1). This band was not 160 present in samples from the negative control strain producing PulG ${ }^{\mathrm{ESA}}$ and the Pul T2SS 161 lacking PulG ( $\Delta \mathrm{G}$, Figure $1 \mathrm{~A}$, lane 2). The intensity of this band was higher in the strain 162 where both plasmids carried the wild type copy of the pulG gene (Figure 1A, lane 3), 163 compared to the strain carrying a $\Delta p u / G$ version of T2SS complemented with wild type pulG 164 (Figure 1A, lane 4). To test whether PulG ${ }^{\mathrm{EA}}$ was co-assembled with $\mathrm{PulG}^{\mathrm{WT}}$, the gel slices corresponding to the $14.5-\mathrm{kDa}$ band were excised from lanes 1 (strain producing PulG ${ }^{\mathrm{WT}}$ and $\mathrm{PuIG}^{\mathrm{EFA}}$ ) and 4 (producing PulG ${ }^{\mathrm{WT}}$ ) and analysed for protein content using top-down mass spectrometry (analysis of intact proteins without any digestion) (Gault et al., 2014; Gault et al., 2015). For PulGWT sample (Figure 1B), two major peaks were found at 14607.56 and 14621.54 Da, corresponding to the molecular mass of PulG (theoretical Mr: $14607.51 \mathrm{Da}$ ) and methylated PulG (theoretical Mr: $14621.53 \mathrm{Da}$ ). For the PulG ${ }^{\mathrm{WT}}+\mathrm{PuIG}^{\mathrm{ESA}}$ sample

171 (Figure 1C), two supplementary peaks corresponding to PulG ${ }^{\text {E5A }}$ (experimental: 14549.49 Da; theoretical: $14549.50 \mathrm{Da}$ ) and methylated PulG ${ }^{\mathrm{EA}}$ (experimental: $14563.53 \mathrm{Da}$; theoretical: $14563.52 \mathrm{Da}$ ) were observed.

To confirm the $\mathrm{N}$-terminal methylation and presence of an alanine residue $(\mathrm{A})$ instead of a Glu (E) at the $5^{\text {th }}$ position in the sequence, we performed High Energy Collision 176 Dissociation (HCD) fragmentation of the $\left(14^{+}\right)$ions of both methylated PulG and methylated $177 \mathrm{PuIG}^{\mathrm{E5A}}$. The MS/MS spectra are shown in Figure 2. The presence of series of b-type ions in both cases confirms without any ambiguity both the methylation of the $\mathrm{N}$-terminus and the identity of the 5th amino acid.

These results show that $\mathrm{PuIG}^{\mathrm{EFA}}$ can assemble into pili, provided it is co-produced 181 with PulGWT. This suggests that PulG ${ }^{\mathrm{E} A}$ likely co-assembles with native PulG into mixed pili. 182 The presence of methylated and non-methylated forms of both PulG variants in the pili 183 shows further that methylation of pseudopilins is not required for assembly. 
187 With the exception of the PulK subunit, which caps the priming complex, all minor 188 pseudopilins in T2SS have the conserved E5 residue. Given its strong conservation among 189 (pseudo)pilins, we wondered whether E5 was also essential for minor pseudopilin function. 190 To address this question, we introduced E5A substitutions in minor subunits PulH, Pull and 191 PulJ and assessed their ability to support PulA secretion in conditions of low, chromosome192 like levels of pul gene expression. The results of three independent secretion assays are 193 shown in Figures $3 \mathrm{~A}$ and supplementary Figure $\mathrm{S1}$, and their quantification is shown in 194 Figure 3B. Under these conditions, the PulG ${ }^{\mathrm{E} A \mathrm{~A}}$ variant was as defective for PulA secretion as 195 the negative control strain lacking PulG (Figure 3A, lanes 1-6), as shown previously 196 (Nivaskumar et al., 2014), The pulH deletion mutant supported a low level of PulA secretion 197 (Figure $3 \mathrm{~A}$, lanes 11,12) confirming that $\mathrm{PulH}$ is required for efficient secretion in physiological expression conditions (Cisneros et al., 2012a). Surprisingly, PulA secretion in

199 the presence of the $\mathrm{PulH}^{\mathrm{E} 5 \mathrm{~A}}$ variant was nearly abolished, suggesting a dominant negative 200 effect of the pulH $H^{E 5 A}$ allele (Figure 3A, lanes 7-12). In contrast, E5A substitutions in minor 201 pseudopilins Pull and PulJ did not affect their function (Figure 3A, lanes 13-18 and 19-24). 202 Replacing the Met-5 with Glu in variant PulK ${ }^{\mathrm{M} E}$ had no effect on PulA secretion (Figure 3A, 203 lanes 25-30), confirming previous results obtained in conditions of pul gene overexpression 204 (Vignon et al., 2003). Thus, the requirement for E5 appears to be specific to PulG and PulH 205 pseudopilins, which are not part of the priming tip complex and have been implicated in the 206 downstream steps of pseudopilus assembly (Cisneros et al., 2012a).

Pseudopilin interaction network

210 The PulJ-Pull-PulK complex presumably provides a template for $\mathrm{PulH}$ incorporation 211 (Cisneros et al., 2012a), as suggested by the formation of the quaternary complex 212 comprising all minor pseudopilin periplasmic domains in the $P$. aeruginosa Xcp T2SS (Yanez 213 et al., 2008; Korotkov and Hol, 2008; Douzi et al., 2009). However, this complex did not 214 interact with the globular domain of the major pseudopilin XcpT in vitro (Douzi et al., 2009), 
215 leaving the question of the mechanism that couples the initiation and elongation stages of 216 assembly open. Based on the findings of $\mathrm{Hu}$ and collaborators (Hu et al., 2002), it has been 217 proposed that the minor pseudopilin quaternary complex and the major pseudopilin interact 218 instead though their hydrophobic segments (Douzi et al., 2009). To test this possibility and to 219 characterise the pseudopilin interaction network, we employed the bacterial two-hybrid 220 (BAC2H) approach that allows one to study interactions between full-length, membrane221 embedded proteins (Karimova et al., 1998). Using this method we had previously 222 demonstrated interactions of Pull with PulJ and PulK (Cisneros et al., 2012a). In the present 223 study, we fused the T18 and T25 fragments of adenylyl cyclase CyaA from Bordetella 224 pertussis to the $\mathrm{N}$-terminus of all five full-length mature pseudopilins and performed $\mathrm{BAC} 2 \mathrm{H}$ assays (Experimental Procedures). Using T18-PulG as bait, we identified strong interactions 226 with two minor pseudopilins, PulH and PulJ (Figure 4). When T18-PulH was used as bait, a 227 specific interaction was observed only with T25-PulG. Although both T18-PulH and T25-PulH 228 chimera consistently showed a tendency to interact with PulJ, these interactions were not 229 statistically significant despite low $P$ values (0.106 and 0.201 , respectively) (Figure $4 A$ and 230 Supplementary Dataset 1). The T18-Pull chimera showed specific interactions with PulJ and

231 PulK (Figure 4), in agreement with previous studies in several T2SSs (Cisneros et al., 2012a; 232 Korotkov and Hol, 2008; Douzi et al., 2009). Conversely, T18-PulJ interacted with Pull 233 (Figure 4A). In addition, T18-Pull, T18-PulJ and T18-PulK showed a weak interaction with 234 PulG. Of note, only PulG formed homo-dimers in the BAC2H assay, consistent with the 235 unique ability of major pseudopilins to form long homo-polymers (Durand et al., 2005). The 236 results of these analyses, summarised schematically in Figure 4B, reveal multiple novel 237 contacts between PulG and minor pseudopilins. Overall, the data are compatible with the 238 formation of complexes containing all five pseudopilin species.

$240 \quad$ Pseudopilin interactions with IM assembly platform components 
242 Previous functional studies suggest that the minor pseudopilin complex primes fibre 243 assembly and that it might activate the ATPase PulE that is associated with the assembly 244 platform (AP) complex (Cisneros et al., 2012a). The AP is essential for T2SS function and 245 contains multiple copies of IM proteins PulC, PulF, PulL and PulM (Py et al., 2001; Possot et 246 al., 2000). To understand the physical and functional link between AP and the pseudopilin 247 complex and to elucidate molecular details of the initiation and elongation steps of assembly, 248 we assessed interactions between the pseudopilins and membrane-embedded AP 249 components by using the $\mathrm{BAC} 2 \mathrm{H}$ system. Therefore, we fused the T18- and T25- CyaA 250 fragments to the N-terminal ends of full-length AP components PulC, PulF, PulL and PulM 251 (Experimental Procedures). In the BAC2H assays, both T18-PulG and T25-PulG interacted 252 specifically with the corresponding PulM- and PulF-CyaA chimera, but not with PulC253 chimera. T18-PulG interacted very weakly with the T25-PulL, but the reciprocal pair did not 254 interact (Figure 5A), which was surprising, given that their homologues in $V$. cholerae, EpsL 255 and EpsG, interact in a cross-linking study (Gray et al., 2011). We confirmed that this was 256 not due to non-functional PulL chimera, since the latter did interact with corresponding PuIM 257 hybrids (Figure 5A, black bars on the right), as expected based on previous studies (Possot 258 et al., 2000; Buddelmeijer et al., 2006). The results, summarized in Figure 5B, revealed strong and specific interactions of PulG with the PulM and PulF components of the assembly 260 platform, suggesting their direct role in pseudopilus elongation. Among the minor pseudopilins, PulH also interacted with PulF and PulM, although 262 these interactions seem weaker than those observed between PulG and PulF or PulM, 263 based on beta-galactosidase activity levels (Figure 5A). A weak "one-way" interaction was 264 also detected between T18-PulK and T25-PulM chimera. In addition, PuIF interacted weakly 265 but significantly with Pull, PulJ and PulK in one out of two protein hybrid pairs. The high beta266 galactosidase activity of strains producing T18-PulF and T25-PulF chimera in the BAC2H 267 system (Figure 5A) shows a strong propensity of PulF for dimer formation. 
Given the suggested role of E5A in an early step of the assembly process (Campos et al., 2010; Nivaskumar et al., 2014), we asked whether the E5A mutation affects interactions of PulG with one of the assembly factors. In BAC2H assay the PulG ${ }^{\mathrm{E} A}$ variant showed equal or stronger interaction signal with PulF, compared to native PulG, as indicated by the high beta-galactosidase activities of T18-PulG ${ }^{\mathrm{E} 5 \mathrm{~A}}$ or T25-PulG ${ }^{\mathrm{EAA}}$ hybrids (Figure $6 \mathrm{~A}$ ). In contrast,

276 the same PulG ${ }^{E 5 A}$ hybrids showed a significantly decreased interaction with PulM (Figure $2776 \mathrm{~A})$. These results suggest that mutation E5A specifically alters the interface between PulG and PulM. Likewise, while the E5A substitution in PulH did not affect the PulH-PulF interaction, it reduced the interaction with PulM, supporting a role of E5 in PulM binding (Figure 6B).

The PulG-PulM interaction data were further validated with native, full-length proteins.

282 We analysed PulG interactions with PulM and the effect of the PuIG E5A substitution using a 283 co-purification approach. PulG-His 6 , PulG ${ }^{\mathrm{EA}}-\mathrm{His}_{6}$ or $\mathrm{PuIG}^{\mathrm{WT}}$ were co-produced with PulM in 284 the absence of other T2SS components (Experimental Procedures). Membrane proteins were extracted from these strains with Triton-X-100 and separated on the Ni-NTA columns. PulM was co-eluted with PulG-His 6 (Figure 7A, lanes 6-11). Despite the higher levels of the

$\mathrm{PuIG}^{\mathrm{E5A}}-\mathrm{His}_{6}$ variant produced and retained on the column, the quantity of co-purified PulM 288 was significantly reduced (Figure 7A, lanes 17-22). Quantification of the relative amounts of 289 PulG and PulM shows that the amount of co-eluted PulM was reduced nearly ten-fold, 290 relative to PulG-His 6 (Figure 7B). As shown in Figure 7A, PulG and PulM were present in 291 very low amounts in the elution fractions when membrane proteins were extracted from the 292 control strain producing untagged PuIG (lanes 28-33), indicating very low nonspecific binding 293 to the Ni-NTA resin.

294 The results of these affinity co-purification experiments were in good agreement with 295 those of the BAC2H analysis, revealing specific binding of PulM to PulG in vivo. They confirm 296 that the E5A substitution in PulG, which abolishes pseudopilus assembly and protein 297 secretion, specifically affected the interaction between PulG and PulM. 


\section{Discussion}

301 Archaeal pili and flagella, as well as bacterial T2SS, T4P and competence systems use similar machineries to assemble helical fibres from membrane-anchored (pseudo)pilin

303 subunits, suggesting a common mechanism. The globular (pseudo)pilin domains, exposed 304 on the fibre surface, show variability in size and sequence, presumably optimized to promote 305 specific functions (Berry and Pelicic, 2014; Nivaskumar and Francetic, 2014; Shahapure et 306 al., 2014). In contrast, their hydrophobic segments are highly conserved, which might reflect 307 constraints imposed by their biogenesis and assembly pathway (Campos et al., 2013). For 308 example, high hydrophobicity is key for efficient targeting to the SRP and Sec pathways 309 during integral membrane protein biogenesis (Francetic et al., 2007; Arts et al., 2007) and 310 additional constraints might be linked to specific interactions with the prepilin peptidase or 311 with fibre assembly factors. Here we used the Klebsiella T2SS as a model to study 312 pseudopilus assembly, focusing on the most conserved (pseudo)pilin residue E5 playing an 313 essential role in this process.

314 Although the E5 residue is involved in pseudopilus stabilisation via long-range 315 interactions, genetic data suggest it plays a critical role at a step preceding pseudopilin 316 docking. Using two complementary mass spectrometry methods we demonstrated 317 unambiguously that PulG ${ }^{\mathrm{EFA}}$ co-assembles with PulG ${ }^{W T}$ into mixed PulG pili containing the 318 methylated and non-methylated forms of PuIG and PuIG ${ }^{E 5 A}$. This confirms previous findings 319 that E5A substitution does not prevent N-methylation of PulG (Pugsley, 1993) and shows 320 that methylation is not essential for pseudopilus assembly. This is in agreement with 321 assembly of T4P in the methylase-deficient pilD mutants of $P$. aeruginosa (Pepe and Lory, 322 1998) and with the small amount of non-methylated PulG detected in T2SS pilus 323 preparations (Köhler et al., 2004). Compared to PulG ${ }^{W T}$, the degree of N-methylated PulG ${ }^{\mathrm{E} A}$ 324 was lower, which might be a consequence of pulG overexpression increasing the load on the 325 methyl-transferase function of the prepilin peptidase PulO. Of note, the proportion of PulG ${ }^{\mathrm{EFA}}$ 
326 in the mixed pili was substantially lower than $50 \%$, which could be explained by the 327 dependence on $\mathrm{PuG}^{\mathrm{WT}}$ for assembly, as well as by the predicted impact of the E5A 328 substitution on fibre stability, due to the loss of long-range interactions with $\mathrm{K}_{2} 8^{\mathrm{P}-3}$ and $\mathrm{K} 35^{\mathrm{P}-3}$ 329 (Nivaskumar et al., 2014). We propose that co-assembly of PulG ${ }^{\mathrm{EFA}}$ relies on its ability to 330 form heterodimers with PulG ${ }^{\mathrm{WT}}$ via the previously characterised electrostatic contacts of 331 conserved residues E44 and D48 of the incoming subunit $\left(\mathrm{P}^{-1}\right)$ with $\mathrm{R} 87$ and $\mathrm{R} 88$ of the $\mathrm{P}$ 332 subunit incorporated in the PulG pilus (Campos et al., 2010; Nivaskumar et al., 2014). This 333 association with PulG ${ }^{W T}$ in the membrane would bypass the defect of PulG ${ }^{\text {E5A }}$ possibly 334 related to its targeting to the assembly site, as discussed below.

Pseudopilus assembly requires the formation of the PulJ-Pull-PulK priming complex, essential for protein secretion by the T2SS (Cisneros et al., 2012a). While our previous studies demonstrated the correlation between the formation of this complex and pseudopilus 338 assembly activation (Cisneros et al., 2012a; Cisneros et al., 2012b), the molecular events 339 linking the two processes have not been identified. To understand this link, we employed the 340 BAC2H approach and characterised the network of interactions between full-length 341 membrane-embedded pseudopilins and between individual pseudopilins and the AP 342 components. The results confirmed the Pull-PulJ and Pull-PulK interactions observed in vitro 343 in T2SSs from Vibrio (Yanez et al., 2008), E. coli (Korotkov and Hol, 2008) and $P$. 344 aeruginosa (Douzi et al., 2009). In the BAC2H assay, the full-length PulH showed only a 345 weak tendency to interact with PulJ, although the periplasmic domains of their Pseudomonas 346 homologues interacted in vitro (Douzi et al., 2009). In addition, contrary to the latter study, we 347 identified direct contacts of PulG with $\mathrm{PulH}$, which are in agreement with the results obtained 348 in Xanthomonas T2SS studies ( $\mathrm{Hu}$ et al., 2002). In the $P$. aeruginosa T4P, the BAC2H 349 analysis revealed very similar interactions between FimU, the PulH structural homologue, 350 and the major pilin PilA, showing that these contacts are conserved in both systems (Nguyen 351 et al., 2015). Morover, in the same study, FimU also failed to interact with FimW, the PulJ 352 equivalent in this T4P system. Finally, we also identified novel direct contacts of PulG with 353 PulJ that are in good agreement with the proposed structural models wherein $\mathrm{GspH}$, but also 
354 GspG, could be docked directly to GspJ at the base of the tip complex (Korotkov and Hol, 355 2008). An interaction between PulG and PulJ might bypass the requirement for PulH, 356 explaining the minor effect of pulH deletion on PulG pilus assembly (Cisneros et al., 2012a) 357 and on PulA secretion (Sauvonnet et al., 2000) in conditions of pul gene overexpression. 358 However, when the T2SS components are not overproduced, this requirement becomes 359 more critical, consistent with the strongly defective PulA secretion in the absence of PulH 360 (Figure 3). In addition, all minor pseudopilin subunits showed a weak tendency to interact 361 with PulG in the BAC2H assay, which might explain the capacity of Pull-PulJ and Pull-PulK 362 minor pseudopilin pairs to partially restore initiation of PuIG assembly in $\Delta p u l H I J K$ mutants 363 (Cisneros et al., 2012a).

364 How do pseudopilins connect to the AP and with the associated ATPase, essential for 365 fibre elongation? The results of our BAC2H analysis show that PulH and PulG, but not the 366 other minor pseudopilins, interact specifically with two AP components, PuIM and PuIF, 367 which might provide a basis for the recruitment of the initiation complex to the assembly site.

368 We demonstrated that PulG and PulH interact with the conserved AP component PulF, 369 presumably via TM segments of the two partners, given the absence of prominent 370 periplasmic segments in PulF. In view of their high conservation, PulF and its equivalents in 371 T4P assembly systems might be the main factors imposing restraints on the pilin primary 372 sequence. The sequence conservation of pseudopilin TM segments is consistent with weak 373 but significant binding of all minor pseudopilins to PulF in the BAC2H assay (Figure 5). 374 Although we cannot exclude the possibility that these weak interactions anchor the PulJ-I-K 375 priming complex to PulF, we favour the model wherein the quaternary minor pseudopilin 376 complex binds to PulF via PulH. In addition, PulF showed a strong tendency to form dimers 377 in this assay, consistent with the crystallography data (Abendroth et al., 2009) and with the 378 observed oligomerisation of its homologue PilC from Thermus thermophilus (Karuppiah et al., 2010). In the recent study of the Myxococcus xanthus T4P architecture by cryo-electron 380 tomography, the PuIF homologue PilC also appears to form a dimer (Chang et al., 2016). 381 During fibre elongation, PulF dimers could act as membrane scaffolds or docking sites for the 
382 minor pseudopilin complex (or the nascent pilus) on one hand, and the incoming PulG 383 subunits on the other, to facilitate assembly. In the large-scale BAC2H interaction study of 384 T4P from Neisseria meningitidis, the major pilin PilE interacted with the PulF homologue 385 PilG, and both PilE and PilG formed homodimers, much like PulG and PulF (Georgiadou et al., 2012). In the P. aeruginosa and Myxococcus xanthus T4P, the PulF homologue called 387 PilC interacts with the ATPase PilB (Takhar et al., 2013; Bischof et al., 2016), consistent with 388 its role in transduction of mechanical energy generated by the ATPase. In the E. coli bundle389 forming pili (Bfp) the ATPase activity of BfpD is stimulated by specific regions of the PulF 390 homologue BpfE (Crowther et al., 2005). Importantly, the BAC2H results revealed interactions of PulG and PulH with the PuIM component of the AP. Moreover, the direct interaction of native PulG and PulM was 393 confirmed by a complementary co-purification approach. So far, PulM has been implicated in 394 stabilization of PulL within the AP complex (Possot et al., 2000). While the results of the 395 BAC2H analysis confirm PulM interaction with PulL, widely observed and well-studied in 396 other T2SSs (Py et al., 2001; Johnson et al., 2007; Lallemand et al., 2013), the strong and 397 specific interactions with PulG and PulH reveal a novel role for PulM, possibly in pseudopilin recruitment to the assembly complex. Furthermore, we have shown that E5A substitutions in PulG and PulH interfere with their interactions with PulM in the absence of any other Pul 400 factors, which implies that, in both cases, the E5 residue is a key feature of the PulG-PulM 401 and PulH-PulM interfaces. These interaction defects caused by the E5A substitution likely 402 account for the dramatic assembly defect of PulG ${ }^{\mathrm{EFA}}$ variant. The trans-dominant effect of the 403 pulH $H^{E 5 A}$ allele might be due to the reduced ability of $\mathrm{PulH}^{\mathrm{ESA}}$ to target the priming complex to 404 PuIM, as discussed below. In contrast, residue E5 is dispensable for function of Pull and PulJ 405 subunits, although it might provide optimal packing with the $\mathrm{N}$-termini of the distal 406 neighbouring pseudopilins in the complex, as proposed previously (Craig et al., 2003).

407 Our results show that PulG interacts strongly and specifically with PulM, but only very 408 weakly with PulL, which apparently contradicts the studies in Vibrio, showing that EpsG could 409 be cross-linked with EpsL (Gray et al., 2011). This difference in interaction might reflect 
410 differences between the Vibrio and Klebsiella T2SSs, or could be due to a difference in the

411 techniques used to detect G-L interactions. The interaction of EpsG with EpsL was observed 412 using chemical cross-linking, which might enhance and stabilize a weak or indirect 413 association (Gray et al., 2011). Here, we identified direct associations of PulG with PulM and 414 PuIF in the absence of other T2SS components using BAC2H, and also, in the case of PulG and PuIM, by co-purification in the presence of nondenaturing detergents. Nevertheless, both 416 studies support a similar model wherein interactions of major pseudopilin with the L-M 417 complex result in pilin recruitment to the assembly site.

418 The architecture of the T2SS, proposed based on partial high- and low-resolution 419 structural data of individual components (McLaughlin et al., 2012) is very similar to that of the Thermus thermophilus or Myxococcus xanthus T4P determined by cryo-electron tomography

421 (Gold et al., 2015; Chang et al., 2016). In all models, the secretin and the AP components 422 homologous to PulC, PulL and PulM delimit a cage-like compartment that encloses PulF 423 within and connects directly with the ATPase PulE at its base. While cryoEM tomography 424 shows that the pilus occupies the interior of this compartment, PilO (a PulM homologue) and 425 its interacting partner PilN (corresponding to the transmembrane and periplasmic domains of 426 PulL) were placed in the lower periplasmic ring surrounding the cavity (Chang et al., 2016). Integrated with the previous data, the results of this study allow us to propose a 428 schematic working model of pseudopilus assembly initiation and elongation (Figure 8). In this 429 model, one of the initial steps, following pseudopilin maturation by the prepilin peptidase, 430 would be the assembly of the priming complex PulJ-Pull-PulK and its binding to PulH and 431 PulG (step 1). Consistent with the direct binding of PulH and PulG to PulF, and with PulF 432 self-interaction, this complex would associate with a dimer of PulF (step 2). In step 3, the 433 pseudopilins and PuIF would bind to PulM, outside or within the pre-assembled complex that 434 includes the secretin PuID channel in the OM bound to the IM components PulC and PulL. 435 An independent assembly of this latter complex is consistent with the results of cellular 436 localisation studies of T2SS components fused to fluorescent protein tags (Buddelmeijer et 437 al., 2009; Lybarger et al., 2009), as well as with the T4P cryo-electron tomography data 
438 (Chang et al., 2016). Importantly, association of PulM with PulF-pseudopilin complex 439 requires residue E5 of PulG and PulH (Figure 8, step 3). Results of the T4P study by cryo440 tomography suggest that the ATPase is recruited to the assembly site in the final step of the 441 T4P complex biogenesis (Chang et al., 2016). A similar order of assembly, in which the 442 incorporation of the PulF-pseudopilin complex into the incomplete T2S machine would 443 precede the recruitment of the ATPase PulE, is possible in T2SS (steps 4 and 5). However, 444 there is an important difference between the two systems at the level of PulL, which 445 corresponds to a fusion between PilM and PilN of T4P, leaving open a possibility that 446 ATPase recruitment occurs independently of PulF.

447 During pseudopilus elongation, PulG, possibly in the form of dimers, would be 448 recruited to the assembly site via interaction with PulM, through the critical residue E5 (step 449 6). PulG would dock to the available PulF "acceptor" site and bind to the membrane450 embedded $\mathrm{PulG}^{\mathrm{P}+1}$ subunit incorporated in the pseudopilus. ATP hydrolysis could drive 451 rotation of the pilus via PulF to spool the protomer $P$ into the growing fibre (step 7), following 452 the one-start helix assembly model that we proposed and described earlier (Campos et al., 453 2010; Nivaskumar et al., 2014). Fibre elongation would comprise cycles of targeting, docking 454 and spooling events (steps 6 to 9 ).

Based on this model, we propose that co-assembly of PuIG ${ }^{\mathrm{EFA}}$ relies on its ability to 456 form heterodimers with $\mathrm{PulG}^{\mathrm{WT}}$, which would promote targeting to the assembly site 457 (Campos et al., 2010; Nivaskumar et al., 2014). The trans-dominant effect of PulH ${ }^{\mathrm{ESA}}$ variant 458 would be due to its association to pseudopilin-PulF complex but not to PulM. While providing 459 testable hypotheses, this model raises numerous questions, notably those related to the 460 mechanism of exoprotein substrate recruitment and transport. Whether a recruitment step 461 accompanies uptake of exoprotein substrate molecules, or whether there are mechanisms 462 that control substrate entry to the preformed assembly site, are only some of the questions 463 that need to be addressed by further investigation.

464 The interaction network of pseudopilin subunits and assembly platform proteins in the 465 inner membrane described here will provide the basis for further biochemical and structure 
466 function analysis of pseudopilus biogenesis and its mechanistic link with T2SS-mediated 467 protein secretion.

468

\section{Experimental Procedures}

470

471

472

473

474

475

476

477

478

479

480

481

482

483

484

485

486

487

488

489

490

491

492

\section{Bacterial strains and plasmids}

The Escherichia coli strain DH5 $\alpha\left[F^{\prime}\right.$ lacl ${ }^{Q} \Delta$ lacZM15 pro+ Tn10)] was used for recombinant DNA experiments. Strains PAP7460 [ $\Delta$ (lac-argF)U169 araD139 relA1 rpsL150 $\Delta$ malE444 malG501] and PAP5207 [ $\Delta$ (lac-argF)U169 araD139 relA1 rpsL150 pcnB::Tn10 (F' lacl ${ }^{Q}$ $\Delta$ lacZM15 pro+)] were used for pul gene expression studies. The $\Delta c y a$ strain DHT1 (Dautin et al., 2000) was used for bacterial two-hybrid experiments. The bacteria were grown in LB medium supplemented with antibiotics, as required: chloramphenicol $(\mathrm{Cm})\left(25 \mu \mathrm{g} \mathrm{ml^{-1 }}\right)$, ampicillin (Ap) $\left(100 \mu \mathrm{g} \mathrm{ml}^{-1}\right)$ or kanamycin $(\mathrm{Km})\left(15\right.$ or $\left.25 \mu \mathrm{g} \mathrm{ml}^{-1}\right)$. The expression of the pul genes was induced by addition of $0.4 \%$ maltose. Isopropyl- $\beta$-D-thiogalactoside was added to induce lacZ promoter-controlled gene expression in strain PAP5207.

\section{Plasmid construction}

Plasmids used in this study are listed in Table 1. Plasmid DNA purification, gel extraction and PCR product purification were performed using appropriate Qiagen kits. Restriction enzymes, DNA ligase and other molecular biology reagents were purchased from Fermentas or New England Biolabs. The high-fidelity Pwo polymerase (Roche) was used for PCR amplification and site directed mutagenesis using the modified Quick-change method. The list of oligonucleotides (synthesized by Sigma Genosys) used for cloning or site-directed mutagenesis is provided in Supplementary Table 1. To construct the BAC2H chimera, the pul genes were PCR-amplified using corresponding primers, treated with Dpnl and cloned into 
493 pKT25 and pUT18c vectors using Kpnl and EcoRI enzymes. All plasmids were sequenced 494 by GATC.

498 Competent cells of strain DHT1 were co-transformed with pUT18C and pKT25 derivatives 499 and bacteria were grown for $48 \mathrm{~h}$ at $30^{\circ} \mathrm{C}$ on LB plates containing $\mathrm{Ap}$ and $\mathrm{Km}$. Colonies were 500 picked at random and inoculated into $5 \mathrm{ml}$ cultures in LB containing $\mathrm{Km}$ and $\mathrm{Ap}$, grown 501 overnight and inoculated the next day into fresh medium containing $1 \mathrm{mM}$ IPTG. Bacteria 502 were cultured to mid-log phase and $\beta$-galactosidase activity was measured as described 503 (Miller, 1972). At least 2 independent experiments were performed with 3 randomly picked 504 transformants. Mean values were presented by bar graphs, and error bars indicate standard 505 deviation. Microsoft Excel software was used for data processing and presentation. The 506 statistical analysis was performed using the non-parametric Kruskal-Wallis test, followed by 507 Dunn's post-test for multiple comparisons, using the Prism software.

Purification of PulG pili

511 PAP7460 bacteria were transformed with pCHAP8185 plasmid containing all pul T2SS 512 genes including $p u / G^{W T}$, or its derivative pCHAP8184 lacking pulG, and with pSU18 or its 513 derivatives pCHAP1205 (PulG ${ }^{W T}$ ) or pCHAP7790 (PulG ${ }^{\mathrm{EFA}}$ ) carrying different pulG alleles 514 under control of the lacZ promoter. Bacteria were cultured on LB agar containing Ap, Cm and $5150.4 \%$ maltose. After incubating for $48 \mathrm{~h}$ at $30^{\circ} \mathrm{C}$, bacteria were harvested and resuspended 516 in LB medium. Pili were sheared by vortex treatment and 20 passages through a 26 -Gauge 517 needle. Two consecutive centrifugation steps at $16000 \mathrm{~g}$ for $20 \mathrm{~min}$ were used to separate 518 bacteria and pili. The pilus fractions were further collected by ultracentrifugation in rotor Ti60 519 at $150000 \mathrm{~g}$ and pili were resuspended in $20 \mathrm{mM} \mathrm{HEPES} \mathrm{for} \mathrm{further} \mathrm{analysis} \mathrm{by} \mathrm{SDS-PAGE}$ 520 and mass spectrometry. 
524 All samples were desalted by $\mathrm{C}_{4}$ ZipTip® (Millipore) and eluted directly into a $10 \mu \mathrm{L}$ spray 525 solution of methanol:water:formic acid (75:25:3). Between 2 and $6 \mu \mathrm{L}$ were introduced into 526 an Orbitrap Velos mass spectrometer, equipped with ETD module (Thermo Fisher Scientific, 527 Bremen, Germany) using a TriVersa NanoMate® (Advion) in positive ion mode. The spray 528 voltage was set to $1.2-1.6 \mathrm{kV}$ and back-pressure to $0.3-0.4$ psi. A full set of automated 529 positive ion calibrations was performed immediately prior to mass measurement. The 530 transfer capillary temperature was lowered to $100^{\circ} \mathrm{C}$, sheath and auxiliary gasses switched 531 off and source transfer parameters optimised using the auto tune feature. Helium was used 532 as the collision gas in the linear ion trap. The FT automatic gain control was set at $1 \times 10^{6}$ for

533 MS experiments. Spectra were acquired in the FTMS in full profile mode with between 10 534 microscans over several minutes, with averaging on and set to max, and a resolution of 53560,000 at $400 \mathrm{~m} / \mathrm{z}$. The final few spectra were then averaged using Qualbrowser in Thermo 536 Xcalibur 2.1 and deconvoluted using Xtract to produce zero charge mass spectra.

537 For MS/MS experiments, the FT automatic gain control was set at $2 \times 10^{5}$. Ions corresponding 538 to the isotopic distribution of a single charge state $\left(14^{+}\right)$were selected with the largest 539 possible window to avoid overlap with neighbouring species but minimize signal loss. HCD 540 was performed at $27 \mathrm{eV}$ and spectra were acquired in the FTMS in full profile mode at a 541 resolution of 60,000 at $400 \mathrm{~m} / \mathrm{z}$, with 10 microscans and with averaging on and set to the 542 maximum value. The final few spectra were then averaged using Qualbrowser in Thermo 543 Xcalibur 2.1 and deconvoluted using Xtract to produce singly charged MS/MS spectra. $544 \mathrm{MS} / \mathrm{MS}$ spectra were interpreted manually. 
548 Proteins from bacterial extracts were separated by electrophoresis on $9 \%$ or $10 \%$ 549 polyacrylamide gels and transferred onto nitrocellulose membranes (ECL, Amersham) using 550 a semi-dry blotting apparatus. Membranes were blocked with 5\% milk in TBST (10 mM Tris, $551150 \mathrm{mM} \mathrm{NaCl}, 0.05 \%$ Tween 20) and incubated with polyclonal antisera raised against 552 purified PulA, PulG and MalE-PulM (diluted 1:1000 in TBST-5\% milk), followed by four 10-

553 min washes and incubation in horseradish peroxidase-coupled anti-rabbit antibody (1:40,000;

554 Amersham). Membranes were developed by enhanced chemiluminescence ECL2 (Thermo) 555 or Western Lightning Plus ECL (PerkinElmer) and recorded using the Typhoon phosphor556 imager (GE) or LAS 4000 imager (Fujifilm). ImageJ software (Abramoff et al., 2004) was 557 used to quantify the density of bands.

558

PulA secretion assay

560

561 PAP5207 bacteria were transformed with pCHAP8185 plasmid derivatives containing pul 562 genes with corresponding pseudopilin gene knockouts, and a pSU18 empty vector or its 563 derivatives containing the missing wild type or mutant pseudopilin gene (Table 1). Bacteria 564 were grown in LB containing $\mathrm{Ap}$ and $\mathrm{Cm}$ in the presence of $0.4 \%$ maltose, $1 / 10$ volume of $\mathrm{M} 9$ 565 salts and $1 \mathrm{mM}$ IPTG to early stationary phase $\left(\mathrm{OD}_{600 \mathrm{~nm}}>2\right)$. Cultures were normalized to $566 \mathrm{OD}_{600 \mathrm{~nm}}=1$ and bacteria were pelleted by centrifugation for $10 \mathrm{~min}$ at $16000 \mathrm{~g}$ at $4^{\circ} \mathrm{C}$. 567 Bacteria were resuspended in SDS-sample buffer at final concentration of $1 \mathrm{OD}_{600 \mathrm{~nm}}$ per $\mathrm{ml}$. 568 Supernatant fractions were centrifuged again for 10 min and mixed with equal volume of $2 x$ 569 SDS-sample buffer. Samples corresponding to $0.05 \mathrm{OD}_{600 \mathrm{~nm}}$ from each fraction were 570 separated by SDS-PAGE on 9\% Tris-Tricine gels. Proteins were transferred to nitrocellulose 571 membranes (ECL Amersham) by semi-dry electro-transfer. Polyclonal antisera were used for 572 immuno-detection of PulA. Co-purification of PulG-bound proteins 
576 Bacteria of strain PAP7460 producing PulG-His 6 , PulG ${ }^{\mathrm{ESA}}-\mathrm{His}_{6}$ or $\mathrm{PuIG}^{\mathrm{WT}}$ variants encoded 577 by pCHAP1362, pCHAP7785 and pCHAP8658, respectively and PulM (from plasmid 578 pCHAP2393) were cultured at $30^{\circ} \mathrm{C}$ in $\mathrm{LB}$ medium containing $\mathrm{Cm}$ and $\mathrm{Ap}$ to $\mathrm{OD}_{600 \mathrm{~nm}}=1$. 579 Bacteria were collected by centrifugation and broken by sonication in cold TBS (20 mM Tris$580 \mathrm{HCl}, \mathrm{pH} 7.4,150 \mathrm{mM} \mathrm{NaCl}$ ) with $100 \mu \mathrm{g} \cdot \mathrm{ml}^{-1}$ of lysozyme. The lysate was cleared by

581 centrifugation to remove unbroken debris. Membranes were collected by ultracentrifugation 582 at $186000 \times \mathrm{g}$ and resuspended in cold TBS, followed by solubilisation with $2 \%$ Triton X-100. 583 Ultracentrifugation was repeated at $150000 \mathrm{~g}$ to remove non-solubilised membranes. Ni-NTA 584 resin beads were washed with $\sim 10$ volumes of TBS and the solubilised membrane fraction 585 was incubated with Ni-NTA resin at $4^{\circ} \mathrm{C}$. The flow-through was collected, followed by seven 586 washes with two column volumes of TBS supplemented with $20 \mathrm{mM}$ imidazole. Proteins were eluted with TBS containing $300 \mathrm{mM}$ imidazole.

\section{Acknowledgments}

591 We thank Evelyne Richet for insightful comments and for the critical reading of the 592 manuscript, Jenny-Lee Thomassin for the help with statistical analysis, Alexandra East and 593 Peter J. Bond for helpful suggestions. We are grateful to all members of the Laboratory of 594 Macromolecular Systems and Signaling and of the Laboratory of Intercellular Communication 595 and Microbial Infections for helpful discussions and friendly support. We thank Gouzel 596 Karimova and Daniel Ladant for strains, plasmids and advice concerning the BAC2H 597 analysis. This work was funded by the ANR FiberSpace grant NANR-14-CE09-0004. MN 598 was a scholar of the Pasteur-Paris University (PPU) International PhD Program. JSM is 599 funded by a fellowship from the Basque Government. JCR and CM acknowledge the DIM 600 MALINF from the lle de France Region for funding the LTQ Orbitrap Velos. The authors 601 declare that they have no conflict of interest.

602

\section{References}


605

606

607

608

609

610

611

612

613

614

615

616

617

618

619

620

621

622

623

624

625

626

627

628

629

630

631

632

633

634

635

636

637

638

639

640

641

642

643

644

645

646

647

648

649

650

651

652

653

654

655

656

Aas, F. E., H. C. Winther-Larsen, M. Wolfgang, S. Frye, C. Løvold, N. Roos, et al., (2007) Substitutions in the N-terminal alpha helical spine of Neisseria gonorrhoeae pilin affect Type IV pilus assembly, dynamics and associated functions. Mol Microbiol. 63: 69-85.

Abendroth, J., M. Bagdasarian, M. Sandkvist and W. G. Hol, (2004a) The structure of the cytoplasmic domain of EpsL, an inner membrane component of the type II secretion system of Vibrio cholerae: an unusual member of the actin-like ATPase superfamily. $J$ Mol Biol 344: 619-633.

Abendroth, J., D. Mitchell, K. Korotkov, T. Johnson, A. Kreger, M. Sankvist, et al., (2009) The three-dimensional structure of the cytoplasmic domains of EpsF from the type 2 secretion system of Vibrio cholerae. J Struct Biol 166: 303-315.

Abendroth, J., P. Murphy, M. Sandkvist, M. Bagdasarian and W. G. Hol, (2005) The X-ray structure of the type II secretion system complex formed by the N-terminal domain of EpsE and the cytoplasmic domain of EpsL of Vibrio cholerae. J Mol Biol 348: 845855.

Abendroth, J., A. E. Rice, K. McLuskey, M. Bagdasarian and W. G. Hol, (2004b) The crystal structure of the periplasmic domain of the type II secretion system protein EpsM from Vibrio cholerae: the simplest version of the ferredoxin fold. J Mol Biol 338: 585-596.

Abramoff, M. D., P. J. Magelhaes andS. J. Ram, (2004) Image Processing with ImageJ. Biophotonics Internatl 11: 36-42.

Aly, K., E. Beebe, C. Chan, M. Goren, C. Sepúlveda, S. Makino, et al., (2013) Cell-free production of integral membrane aspartic acid proteases reveals zinc-dependent methyltransferase activity of the Pseudomonas aeruginosa prepilin peptidase PilD. Microbiologyopen 2: 94-104.

Arts, J., R. van Boxtel, A. Filloux, J. Tommassen and M. Koster, (2007) Export of the pseudopilin XсpT of the Pseudomonas aeruginosa type II secretion system via the signal recognition particle-Sec pathway. J Bacteriol 189: 2069-2076.

Bartolomé, B., Y. Jubete, E. Martinez and F. de la Cruz, (1991) Construction and properties of a family of pACYC184-derived cloning vectors compatible with pBR322 and its derivatives. Gene 102: 75-78.

Berry, J.-L. andV. Pelicic, (2014) Exceptionally widespread filaments composed of type IV pilins: the prokaryotic Swiss Army knives. FEMS Microbiol Rev 39: 134-154.

Bischof, L. F., C. Friedrich, A. Harms, L. Søgaard-Andersen and C. van der Does, (2016) The type IV pilus assembly ATPase PilB of Myxococcus xanthus interacts with the inner membrane platform protein PilC and the nucleotide binding protein PilM. J Biol Chem 291: 6946-57.

Buddelmeijer, N., O. Francetic and A. Pugsley, (2006) Green fluorescent chimeras indicate nonpolar localization of pullulanase secreton components PulL and PulM. J Bacteriol. 188: 2928-2935.

Buddelmeijer, N., M. Krehenbrink, F. Pecorari and Pugsley, A.P. (2009) Type II secretion system secretin PulD localizes in clusters in the Escherichia coli outer membrane. $J$. Bacteriol. 191: 161-168.

Camberg, J. L., T. L. Johnson, M. Patrick, J. Abendroth, W. G. Hol and M. Sandkvist, (2007) Synergistic stimulation of EpsE ATP hydrolysis by EpsL and acidic phospholipids. The EMBO journal 26: 19-27.

Campos, M., D. Cisneros, M. Nivaskumar and O. Francetic, (2013) The type II secretion system - a dynamic fiber assembly nanomachine. Res Microbiol 164: 545-555.

Campos, M., M. Nilges, D. A. Cisneros and O. Francetic, (2010) Detailed structural and assembly model of the type II secretion pilus from sparse data. Proc Natl Acad Sci U S A. 107: 13081-13086.

Chang, Y.-W., Rettberg, L.A., Treuner-Lange, A., Iwasa, J., Sogaard-Andersen, L. and Jensen, G. (2016) Architecture of the type IVa pilus machine. Science 351: aad2001. 
Cisneros, D. A., P. J. Bond, A. P. Pugsley, M. Campos and O. Francetic, (2012a) Minor pseudopilin self-assembly primes type II secretion pseudopilus elongation. The EMBO journal 31: 1041-1053.

Cisneros, D.A., Péhaut-Arnaudet, G. and O. Francetic, (2012b). Heterologous assembly of type IV pili by a type II secretion system reveals the role of minor pilins in assembly initiation. Mol. Microbiol. 84: 805-818.

Costa, T. R. D., C. Felisberto-Rodrigues, A. Meir, M. S. Prevost, A. Redzej, M. Trokter, et al., (2015) Secretion systems in Gram-negative bacteria: structural and mechanistic insights. Nat Microbiol Rev 13: 343-359.

Craig, L., R. Taylor, M. Pique, B. Adair, A. Arvai, M. Singh, et al., (2003) Type IV pilin structure and assembly: X-ray and EM analyses of Vibrio cholerae toxin-coregulated pilus and Pseudomonas aeruginosa PAK pilin. Mol Cell 11: 1139-1150.

Craig, L., N. Volkmann, A. S. Arvai, M. E. Pique, M. Yeager, E. H. Egelman, et al., (2006) Type IV pilus structure by cryo-electron microscopy and crystallography: implications for pilus assembly and functions. Mol Cell. 23: 651-662.

Crowther, L.J.A. Yamagata, L. Craig, J.A. Tainer and M.S. Donnenberg. (2005) The ATPase activity of BfpD is greatly enhanced by zinc and allosteric interactions with other Bfp proteins. J. Biol. Chem. 280: 24839-24848.

Dautin, N., G. Karimova, A. Ullman and D. Ladant, (2000) Sensitive genetic screen for protease activity based on a cyclic AMP signaling cascade in Escherichia coli. $J$ Bacteriol 182: 7060-7066.

Douzi, B., E. Durand, C. Bernard, S. Alphonse, C. Cambillau, A. Filloux, et al., (2009) The $\mathrm{XcpV/Gspl}$ pseudopilin has a central role in the assembly of a quaternary complex within the T2SS pseudopilus. J Biol Chem 284: 34580-34589.

Durand, E., A. Bernadac, G. Ball, A. Lazdunski, J. Sturgis and A. Filloux, (2003) Type II protein secretion in Pseudomonas aeruginosa: the pseudopilus is a multifibrillar and adhesive structure. J Bacteriol 185: 2759-2758.

Durand, E., G. Michel, R. Voulhoux, J. Kurner, A. Bernadac and A. Filloux, (2005) XcpX controls biogenesis of the Pseudomonas aeruginosa XсpT-containing pseudopilus. $J$ Biol Chem 280: 31378-31389.

Francetic, O., N. Buddelmeijer, S. Lewenza, C. A. Kumamoto and A. P. Pugsley, (2007) Signal recognition particle-dependent inner membrane targeting of the PulG pseudopilin component of a type II secretion system. J Bacteriol 189: 1783-1793.

Gault, G., M. Ferber, S. Machata, A.-F. Imhaus, C. Malosse, A. A. Charles-Orszag, et al., (2015) Neisseria meningitidis type IV pili composed of sequence invariable pilins are masked by multisite glycosylation. PLoS pathogens 11: e1005162.

Gault, J., C. Malosse, S. Machata, C. Millien, I. Podglajen, M. Ploy, et al., (2014) Complete posttranslational modification mapping of pathogenic Neisseria meningitidis pilins requires top-down mass spectrometry. Proteomics 14: 1141-1151.

Georgiadou, M., M. Castagnini, G. Karimova, D. Ladant and V. Pelicic, (2012) Large-scale study of the interactions between proteins involved in type IV pilus biology in Neisseria meningitidis: characterization of a subcomplex involved in pilus assembly. Mol Microbiol. 84: 857-873.

Gold, V.A.M.,R. Salzer, B. Averhoff and W. Külhbrandt. (2015) Structure of a type IV pilus machinery in the open and closed state. eLife 10,7554/eLife.07380.

Gray, M. D., M. Bagdasarian, W. G. Hol and M. Sandkvist, (2011) In vivo cross-linking of EpsG to EpsL suggests a role for EpsL as an ATPase-pseudopilin coupling protein in the type II secretion system of Vibrio cholerae. Mol. Microbiol. 79: 786-798.

Hu, N.-T.,Leu, W.-M.,Lee, M.-S., Che, S.-C., Chen, A., Song; Y.-L. and Che, L.-Y., (2002) XpsG, the major pseudopilin in Xanthomonas campestris, pv. campestris forms a pilus-like structure between cytoplasmic and the outer membranes. Biochem J. 365: 205-211.

Johnson, T. L., M. E. Scott and M. Sandkvist, (2007) Mapping critical interactive sites within the periplasmic domain of the Vibrio cholerae type II secretion protein EpsM. $J$ Bacteriol 189: 9082-9089. 
Karimova, G., J. Pidoux, A. Ullmann and D. Ladant, (1998) A bacterial two-hybrid system

713

714

715

716

717

718

719

720

721

722

723

724

725

726

727

728

729

730

731

732

733

734

735

736

737

738

739

740

741

742

743

744

745

746

747

748

749

750

751

752

753

754

755

756

757

758

759

760

761

762

763

764

765 based on a reconstituted signal transduction pathway. Proc. Natl. Acad. Sci. USA 95: 5752-5756.

Karuppiah, V., D. Hassan, M. Saleem and J. P. Derrick, (2010) Structure and oligomerization of the PilC type IV pilus biogenesis protein from Thermus thermophilus. Proteins 78: 2049-2057.

Kohler, R., K. Schafer, S. Muller, G. Vignon, K. Diederichs, A. Philippsen, et al., (2004) Structure and assembly of the pseudopilin PulG. Molecular microbiology 54: 647-664.

Korotkov, K., M. Sandkvist and W. Hol, (2012) The type II secretion system: biogenesis, molecular archtecture and mechanism. Nat Rev Microbiol 10: 336-351.

Korotkov, K. V. and W. G. Hol, (2008) Structure of the GspK-Gspl-GspJ complex from the enterotoxigenic Escherichia coli type 2 secretion system. Nat Struct Mol Biol. 15: 462468.

Korotkov, K. V., T. L. Johnson, M. G. Jobling, J. Pruneda, E. Pardon, A. Heroux, et al., (2011) Structural and functional studies on the interaction of GspC and GspD in the type II secretion system. PLoS pathogens 7: e1002228.

Lallemand, M., F. Login, N. Guschinskaya, C. Pineau, G. Effantin, X. Robert, et al., (2013) Dynamic Interplay between the periplasmic and transmembrane domains of GspL and GspM in the type II secretion system. PloS One 8: e79562.

Lybarger, S.R., T.L. Johnson,M.D. Gray, A.E. Sikora and M. Sandkvist. (2009) Docking and assembly of the type II secretion complex of Vibrio cholerae. J Bacteriol. 191:31493161.

McLaughlin, L., R. Haft and K. Forest, (2012) Structural insights into the Type II secretion nanomachine. Cu Op Struct Biol 22: 208-216.

Miller, J. H., (1972) Experiments in molecular genetics. Cold Spring Harbor Laboratory, Cold Spring Harbor, New York.

Nguyen, Y., S. Sugiman-Marangos, H. Harvey, S. D. Bell, C. L. Charlton, M. S. Junop, et al., (2015) Pseudomonas aeruginosa minor pilins prime type IVa pilus assembly and promote surface display of the PilY1adhesin. J Biol Chem 290: 601-611.

Nivaskumar, M., G. Bouvier, M. Campos, N. Nadeau, X. Yu, E. Egelman, et al., (2014) Distinct docking and stabilization steps of the pseudopilus conformational transition path suggest rotational assembly of type IV pilus-like fibers. Structure 22: 685-696.

Nivaskumar, M. and O. Francetic, (2014) Type II secretion system: a magic beanstalk or a protein escalator. $B B A$ 1843: 1568-1577.

Nunn, D. and S. Lory, (1991) Product of the Pseudomonas aeruginosa gene pilD is a prepilin leader peptidase. Proc Natl Acad Sci U S A 88: 3281-3285.

Parge, H.E., K.T. Forest, M.J. Hickey, D.A. Christensen, E.D. Getzoff and J.A. Tainer, (1995) Structure of the fibre-forming protein pili at 2.6 A resolution. Nature 378: $32-38$.

Pasloske, B. L. and W. Paranchych, (1988) The expression of mutant pilins in Pseudomonas aeruginosa: fifth position glutamate affects pilin methylation. Mol Microbiol. 2: 489495.

Pasloske, B. L., D. G. Scraba and W. Paranchych, (1989) Assembly of mutant pilins in Pseudomonas aeruginosa: formation of pili composed of heterologous subunits. $J$ Bacteriol 171: 2142-2147.

Possot, O., G. Vignon, N. Bomchil, F. Ebel and A. P. Pugsley, (2000) Multiple interactions between pullulanase secreton components involved in stabilization and cytoplasmic membrane association of PulE. J Bacteriol 182: 2142-2152.

Pugsley, A., (1993) Processing and methylation of PulG, a pilin-like component of the general secretory pathway of Klebsiella oxytoca. Mol Microbiol. 9: 295-308.

Py, B., F. Loiseau and F. Barras, (2001) An inner membrane platform in the type II secretion machinery of Gram-negative bacteria. EMBO Rep. 2: 244-248.

Sandkvist, M., M. Bagdasarian, S. P. Howard and V. J. DiRita, (1995) Interaction between the autokinase EpsE and EpsL in the cytoplasmic membrane is required for extracellular secretion in Vibrio cholerae. The EMBO journal 14: 1664-1673. 
Sauvonnet, N., G. Vignon, A. P. Pugsley and P. Gounon, (2000) Pilus formation and protein secretion by the same machinery in Escherichia coli. EMBO. J. 19: 2221-2228.

Shahapure, R., R. Driessen, M. Haurat, S. Albers and R. Dame, (2014) The archaellum: a rotating type IV pilus. Molecular microbiology 91: 716-723.

Strom, M. and S. Lory, (1991) Amino acid substitutions in pilin of Pseudomonas aeruginosa. J Biol Chem 266: 1656-1664.

Strom, M., D. Nunn and S. Lory, (1993) A single bifunctional enzyme, PilD, catalyzes cleavage and $\mathrm{N}$-methylation of proteins belonging to the type IV pilin family. Proc Natl Acad Sci U S A. 90: 2404-2408.

Takhar, H., K. Kemp, M. Kim, P. L. Howell and L. L. Burrows, (2013) The platform protein is essential for Type IV Pilus Biogenesis. J Biol Chem 288: 9721-9728.

Vignon, G., R. Köhler, E. Larquet, S. Giroux, M. C. Prévost, P. Roux, et al., (2003) Type IVlike pili formed by the type II secreton: specificity, composition, bundling, polar localization, and surface presentation of peptides. J Bacteriol. 185: 3416-3428.

Voulhoux, R., G. Ball, B. Ize, M. V. Vasil, A. Lazdunski, L.-F. Wu, et al., (2001) Involvement of the twin arginine translocation system in protein secretion via the type II pathway. The EMBO journal 20: 6735-6741.

Yanez, M., K. Korotkov , J. Abendroth and W. G. J. Hol, (2008) The Crystal Structure of a Binary Complex of two Pseudopilins: Epsl and EpsJ from the Type 2 Secretion System of Vibrio vulnificus. J. Mol. Biol. 375: 471-486. 
790 Table 1. Plasmids used in this study

\begin{tabular}{|c|c|c|c|}
\hline Name & Origin/resistance & Relevant markers & Source/reference \\
\hline pUT18c & ColE1/Ap ${ }^{R}$ & placZ-T18 & $\begin{array}{l}\text { (Karimova et al., } \\
\text { 1998) }\end{array}$ \\
\hline pKT25 & $\mathrm{p} 15 \mathrm{~A} / \mathrm{Km}^{\mathrm{R}}$ & placZ-T25 & $\begin{array}{l}\text { (Karimova et al., } \\
\text { 1998) }\end{array}$ \\
\hline pUT18c-Zip & ColE $1 / \mathrm{Ap}^{\mathrm{R}}$ & $\begin{array}{l}\text { GCN4 (Leu zipper } \\
\text { region) fused to T18 }\end{array}$ & $\begin{array}{l}\text { (Karimova et al., } \\
\text { 1998) }\end{array}$ \\
\hline pKT25-Zip & $\mathrm{p} 15 \mathrm{~A} / \mathrm{Km}^{\mathrm{R}}$ & $\begin{array}{l}\text { GCN4 (Leu zipper } \\
\text { region) fused to T25 }\end{array}$ & $\begin{array}{l}\text { (Karimova et al., } \\
\text { 1998) }\end{array}$ \\
\hline pSU18 & $\mathrm{p} 15 \mathrm{~A} / \mathrm{Cm}^{\mathrm{R}}$ & placZ, lacZ' & $\begin{array}{l}\text { (Bartolomé et al., } \\
\text { 1991) }\end{array}$ \\
\hline pCHAP1205 & $\mathrm{p} 15 \mathrm{~A} / \mathrm{Cm}^{\mathrm{R}}$ & pSU18 pulG & $\begin{array}{l}\text { (Possot et al., } \\
2000)\end{array}$ \\
\hline pCHAP1329 & $\mathrm{p} 15 \mathrm{~A} / \mathrm{Cm}^{\mathrm{R}}$ & pSU18 pulJ & $\begin{array}{l}\text { (Possot et al., } \\
2000)\end{array}$ \\
\hline pCHAP1331 & $\mathrm{p} 15 \mathrm{~A} / \mathrm{Cm}^{\mathrm{R}}$ & pSU18 pulH & $\begin{array}{l}\text { (Possot et al., } \\
2000)\end{array}$ \\
\hline pCHAP1351 & $\mathrm{p} 15 \mathrm{~A} / \mathrm{Cm}^{\mathrm{R}}$ & pSU18 pull & $\begin{array}{l}\text { (Cisneros et al., } \\
\text { 2012) }\end{array}$ \\
\hline pCHAP1362 & $\mathrm{p} 15 \mathrm{~A} / \mathrm{Cm}^{\mathrm{R}}$ & pSU18 pulG-His ${ }_{6}$ & $\begin{array}{l}\text { (Kohler et al., } \\
\text { 2004) }\end{array}$ \\
\hline pCHAP2393 & ColE1/Ap ${ }^{R}$ & pUC18 pulM & $\begin{array}{l}\text { (Possot et al., } \\
2000)\end{array}$ \\
\hline pCHAP6117 & $\mathrm{p} 15 \mathrm{~A} / \mathrm{Cm}^{\mathrm{R}}$ & pSU18 pulJ ${ }^{E 5 A}$ & This study \\
\hline pCHAP7330 & ColE $1 / A p^{R}$ & pUT18c pulG & $\begin{array}{l}\text { (Nivaskumar et } \\
\text { al., 2014) }\end{array}$ \\
\hline pCHAP7332 & $\mathrm{p} 15 \mathrm{~A} / \mathrm{Km}^{\mathrm{R}}$ & pKT25 pulG & $\begin{array}{l}\text { (Nivaskumar et } \\
\text { al., 2014) }\end{array}$ \\
\hline pCHAP7785 & $\mathrm{p} 15 \mathrm{~A} / \mathrm{Cm}^{\mathrm{R}}$ & pSU18 pulG $^{E 5 A}-H_{i s_{6}}$ & This study \\
\hline pCHAP7790 & $\mathrm{p} 15 \mathrm{~A} / \mathrm{Cm}^{\mathrm{R}}$ & pSU18 pulG ${ }^{E 5 A}$ & This study \\
\hline pCHAP8113 & ColE $1 / A p^{R}$ & pUT18c pulC & This study \\
\hline pCHAP8119 & $\mathrm{p} 15 \mathrm{~A} / \mathrm{Km}^{\mathrm{R}}$ & pKT25 pulC & This study \\
\hline pCHAP8154 & ColE1/Ap ${ }^{R}$ & pUT18c pulM & This study \\
\hline pCHAP8155 & $\mathrm{p} 15 \mathrm{~A} / \mathrm{Km}^{\mathrm{R}}$ & pKT25 puIM & This study \\
\hline pCHAP8184 & ColE1/Ap ${ }^{R}$ & pCHAP8185 $\Delta p u / G$ & $\begin{array}{l}\text { (Campos et al., } \\
\text { 2010) }\end{array}$ \\
\hline pCHAP8185 & ColE1/Ap ${ }^{R}$ & $\begin{array}{l}\text { pulS, pulA } A_{N A} \text { pulB } \\
\text { pulCDEFGHIJKLMNO }\end{array}$ & $\begin{array}{l}\text { (Cisneros et al., } \\
2012 \text { ) }\end{array}$ \\
\hline pCHAP8201 & ColE1/Ap ${ }^{R}$ & $\begin{array}{l}\text { pCHAP8185 } \\
\text { pulH::kan }\end{array}$ & $\begin{array}{l}\text { (Cisneros et al., } \\
\text { 2012) }\end{array}$ \\
\hline pCHAP8209 & ColE $1 / A^{R}$ & pCHAP8185 pulJ::kan & $\begin{array}{l}\text { (Cisneros et al., } \\
\text { 2012) }\end{array}$ \\
\hline pCHAP8212 & ColE1/Ap ${ }^{R}$ & $\begin{array}{l}\text { pCHAP8185 } \\
\text { pulK::kan }\end{array}$ & $\begin{array}{l}\text { (Cisneros et al., } \\
2012 \text { ) }\end{array}$ \\
\hline pCHAP8218 & ColE1/Ap ${ }^{R}$ & pCHAP8185 pull::kan & $\begin{array}{l}\text { (Cisneros et al., } \\
\text { 2012) }\end{array}$ \\
\hline pCHAP8245 & ColE1/Ap ${ }^{R}$ & pUT18c-pull & $\begin{array}{l}\text { (Cisneros et al., } \\
\text { 2012) }\end{array}$ \\
\hline
\end{tabular}




\begin{tabular}{|c|c|c|c|}
\hline pCHAP8246 & ColE1/Ap ${ }^{R}$ & pUT18c pulJ & This study \\
\hline pCHAP8247 & ColE1/Ap ${ }^{R}$ & pUT18c pulK & This study \\
\hline pCHAP8248 & $\mathrm{p} 15 \mathrm{~A} / \mathrm{Km}^{\mathrm{R}}$ & pKT25 pull & $\begin{array}{l}\text { (Cisneros et al., } \\
2012 \text { ) }\end{array}$ \\
\hline pCHAP8249 & $\mathrm{p} 15 \mathrm{~A} / \mathrm{Km}^{\mathrm{R}}$ & pKT25 pulJ & $\begin{array}{l}\text { (Cisneros et al., } \\
2012 \text { ) }\end{array}$ \\
\hline pCHAP8250 & $\mathrm{p} 15 \mathrm{~A} / \mathrm{Km}^{\mathrm{R}}$ & pKT25 pulK & $\begin{array}{l}\text { (Cisneros et al., } \\
2012 \text { ) }\end{array}$ \\
\hline pCHAP8256 & ColE1/Ap ${ }^{R}$ & pUT18c pulH & This study \\
\hline pCHAP8257 & $\mathrm{p} 15 \mathrm{~A} / \mathrm{Km}^{\mathrm{R}}$ & pKT25 pulH & $\begin{array}{l}\text { (Cisneros et al., } \\
2012 \text { ) }\end{array}$ \\
\hline pCHAP8364 & ColE1/Ap ${ }^{R}$ & pUT18c pulF & This study \\
\hline pCHAP8365 & $\mathrm{p} 15 \mathrm{~A} / \mathrm{Km}^{\mathrm{R}}$ & pKT25 pulF & This study \\
\hline pCHAP8418 & $\mathrm{p} 15 \mathrm{~A} / \mathrm{Cm}^{\mathrm{R}}$ & pSU18 pulH & This study \\
\hline pCHAP8420 & $\mathrm{p} 15 \mathrm{~A} / \mathrm{Km}^{\mathrm{R}}$ & pKT25 pulG ${ }^{E 5 A}$ & $\begin{array}{l}\text { (Nivaskumar et } \\
\text { al., 2014) }\end{array}$ \\
\hline pCHAP8434 & $\mathrm{p} 15 \mathrm{~A} / \mathrm{Km}^{\mathrm{R}}$ & pKT25 pulH ${ }^{E 5 A}$ & This study \\
\hline pCHAP8446 & ColE1/Ap ${ }^{R}$ & pUT18c pulH E5A & This study \\
\hline pCHAP8472 & ColE1/Ap ${ }^{R}$ & pUT18c pulL & This study \\
\hline pCHAP8484 & $\mathrm{p} 15 \mathrm{~A} / \mathrm{Km}^{\mathrm{R}}$ & pKT25 pulL & This study \\
\hline pCHAP8568 & $\mathrm{p} 15 \mathrm{~A} / \mathrm{Cm}^{\mathrm{R}}$ & pSU18 pulG & This study \\
\hline pCHAP8639 & $\mathrm{p} 15 \mathrm{~A} / \mathrm{Cm}^{\mathrm{R}}$ & pSU18 pulK ${ }^{\mathrm{M} 5 E}$ & This study \\
\hline pCHAP8670 & ColE1/Ap ${ }^{R}$ & pUT18c pulG ${ }^{E 5 A}$ & $\begin{array}{l}\text { (Nivaskumar et } \\
\text { al., 2014) }\end{array}$ \\
\hline
\end{tabular}




\section{Figure Legends}

A

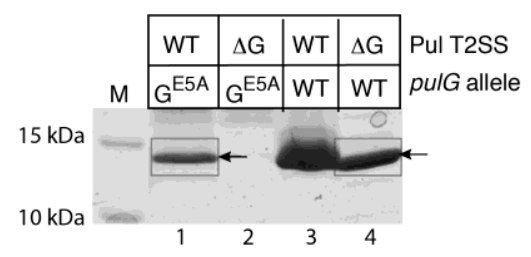

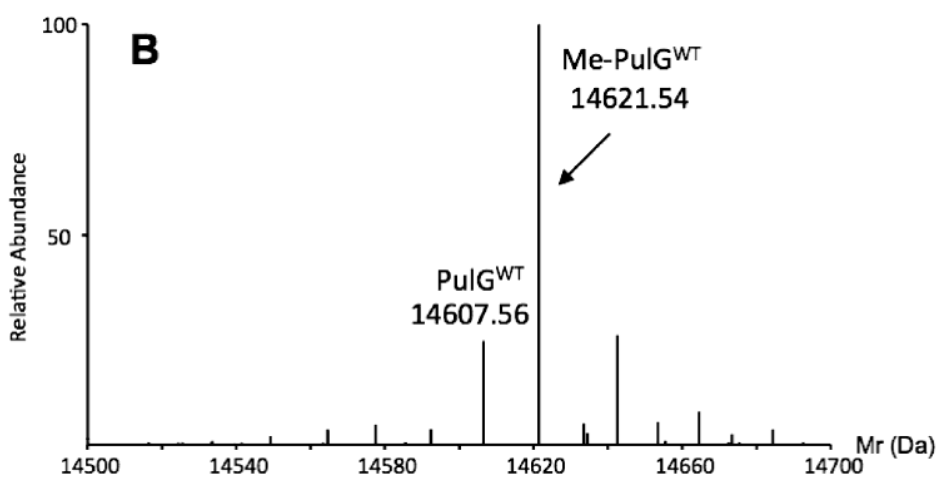

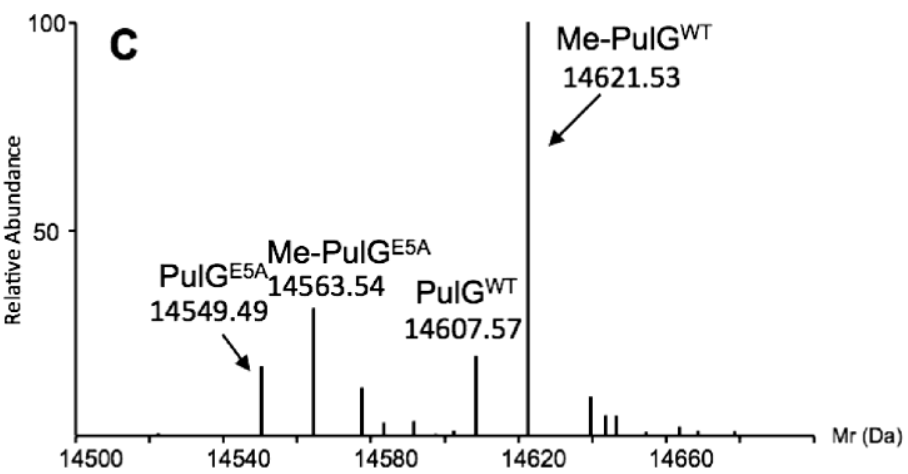

Figure 1. PulG ${ }^{E 5 A}$ is $\mathrm{N}$-methylated and co-assembled into pili with $\mathrm{PuIG}^{\mathrm{WT}}$. A. SDS-PAGE and Coomassie Blue stained sheared fractions from equivalent amounts of bacteria of strain PAP7460 carrying two compatible plasmids (Table 1), as indicated: lane 1, pCHAP8185 (encoding the complete Pul T2SS) and pCHAP7790 (encoding PulG ${ }^{\mathrm{ESA}}$ ); lane 2, pCHAP8184 (encoding Pul T2SS lacking PulG) and pCHAP7790; lane 3, pCHAP8185 and pCHAP1205 (encoding PulG ${ }^{\mathrm{WT}}$ ); and lane 4, pCHAP8184 and pCHAP1205. Only the relevant portions of the gel are shown, with the molecular mass markers indicated on the left. The expected molecular mass of PulG is around $14.6 \mathrm{kDa}$. The squares and arrowheads indicate samples that were further analysed by mass spectrometry. B. Deconvoluted high-resolution mass spectrum (in Mr) obtained for the sample in lane 4 (PAP7460 carrying pulG ${ }^{\mathrm{WT}}$ allele) indicating the presence of a minor peak at 14607.56 Da corresponding to PulG ${ }^{W T}$ and a major peak at $14621.54 \mathrm{Da}$ corresponding to methylated PulG ${ }^{\mathrm{WT}}$; C. Deconvoluted high resolution mass spectrum (in $\mathrm{Mr}$ ) obtained for the sample in lane 1 (PulG ${ }^{\mathrm{WT}}+\mathrm{PulG}^{\mathrm{ESA}}$ ) indicating the presence of supplementary peaks corresponding to PulG ${ }^{\mathrm{E} 5 \mathrm{~A}}(14549.49 \mathrm{Da})$ and methylated PulG ${ }^{\mathrm{EAA}}$ (14563.53 Da). 

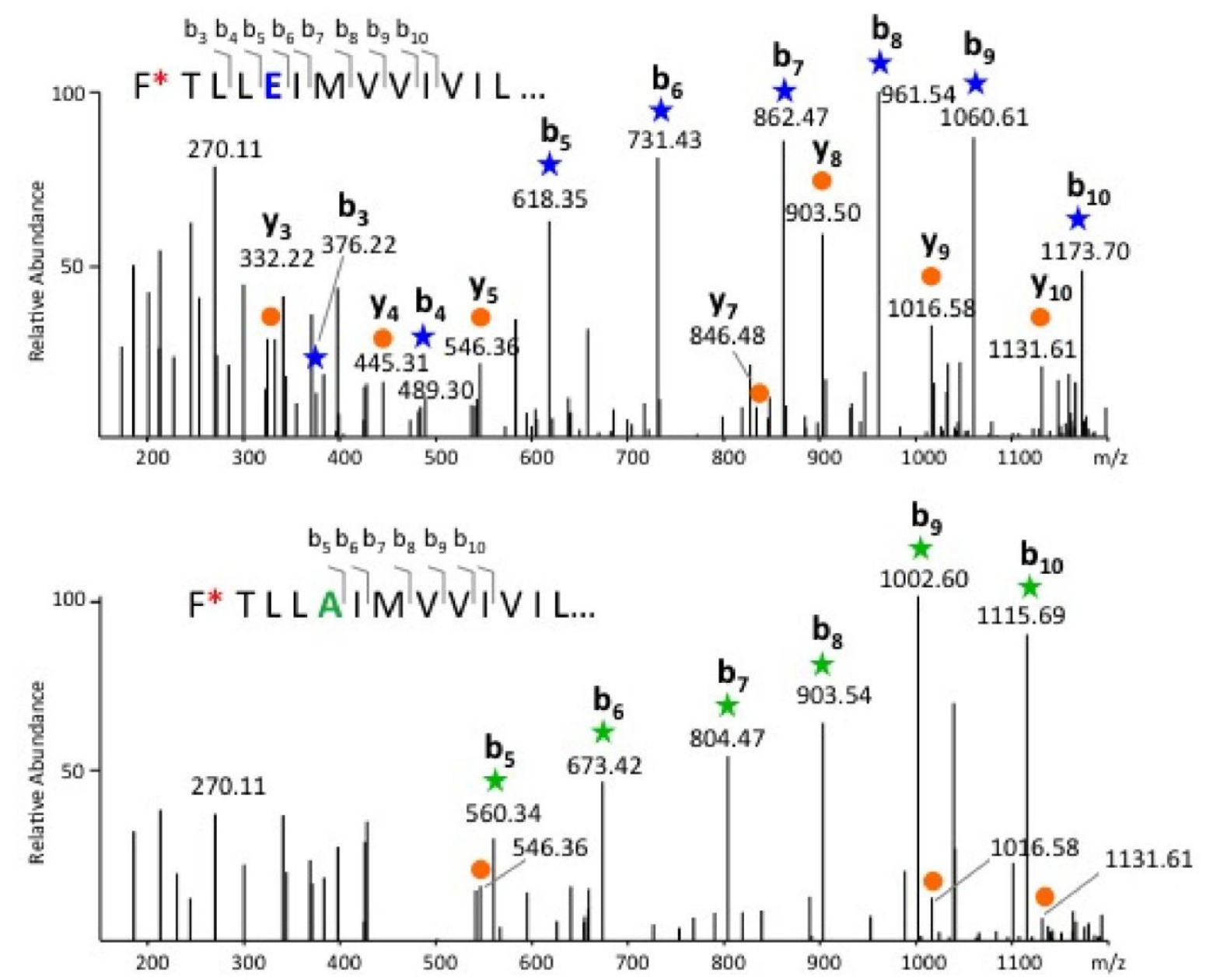

814 Figure 2. High-energy Collision Dissociation (HCD) analysis of methylated PulG ${ }^{W T}$ and 815 PulG $^{\mathrm{ESA}}$ variants co-assembled into pili. HCD fragmentation spectra of the (14+) ion of 816 methylated PulG ${ }^{W T}$ at 1046.05 (top) and methylated PulG ${ }^{\mathrm{ESA}}$ at 1041.83 (bottom). 817 Methylation of the $\mathrm{N}$-terminal residue is indicated with an asterisk. The series of $\mathrm{N}$-terminal $\mathrm{b}$ 818 type ions obtained in both cases and marked with a star unambiguously delineates the 819 presence of an $\mathrm{E}$ at the $5^{\text {th }}$ position in PulG ${ }^{\mathrm{WT}}$ and an $\mathrm{A}$ in $\mathrm{PuIG}^{\mathrm{E}}{ }^{\mathrm{A} A}$. C-terminal $y$-type ions are 820 depicted with a circle. 
A

\begin{tabular}{|l|l|l|l|l|l|l|l|l|l|l|l|l|l|l|}
\hline \multicolumn{2}{|c|}{ PuIG } & \multicolumn{2}{c|}{ PulH } & \multicolumn{3}{c|}{ Pull } & \multicolumn{3}{c|}{ PulJ } & \multicolumn{3}{c|}{ PulK } \\
\hline WT & E5A & - & WT & E5A & - & WT & E5A & - & WT & E5A & - & WT & M5E & - \\
\hline C S C S C S C S C S C S C S C S C S C S C S C S C S C S C S \\
\hline
\end{tabular}

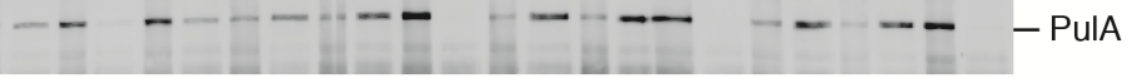

B

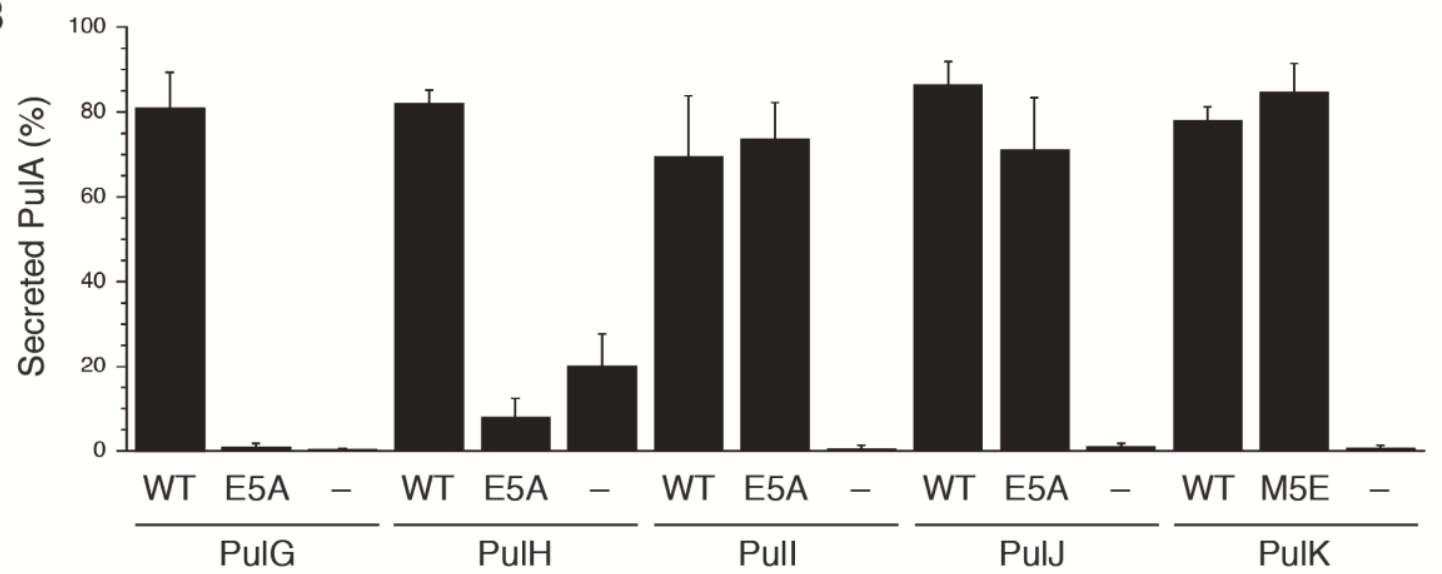

Figure 3. Effect of $5^{\text {th }}$ residue substitutions on pseudopilin function. Pullulanase (PuIA) secretion was assayed in E. coli strain PAP5207 containing pCHAP8185 derivatives with single deletions of pseudopilin genes was complemented with pSU18 plasmid (-) or its derivatives expressing the wild type (WT) or the mutant (E5A or M5E) allele of the missing pseudopilin gene as indicated above the lanes. A. A representative of three independent experiments is shown. Equivalents of $0.05 \mathrm{OD}_{600 \mathrm{~nm}}$ of cell (C) or supernatant (S) fractions were analysed on 9\% Tris-Tricin SDS-PAGE and detected using anti-PulA antibodies. B. Quantification of the fraction of secreted PulA in the three independent experiments shown in panel A and in Figure S1. Bar graphs represent the mean values and the error bars indicate standard deviation. 
A

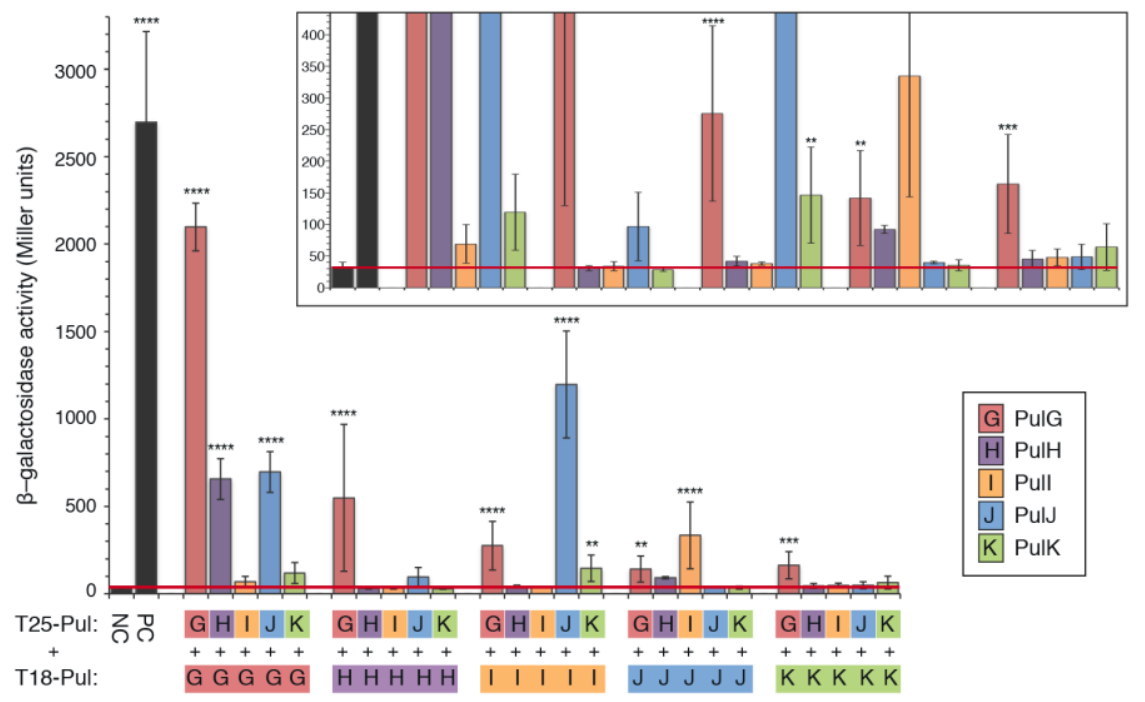

B

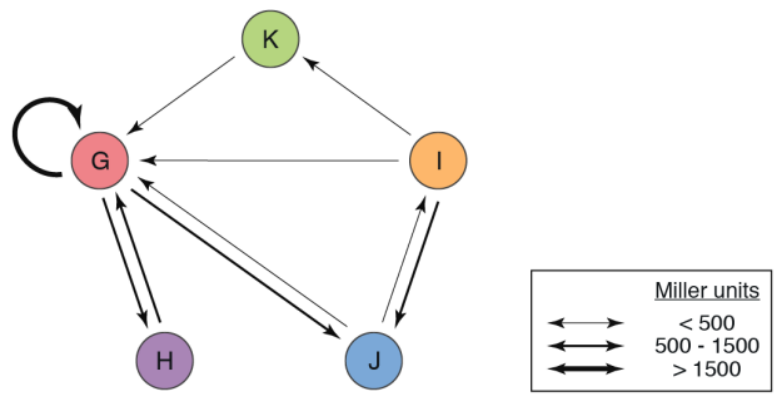

835

836

837

838

839

840

841

842

843

844

845

846

847

848

849

850

851

852

853

854

855

Figure 4. Pseudopilin interaction network determined by the bacterial two-hybrid analysis. A. Beta-galactosidase activities of DHT1 bacteria co-producing indicated T18 and T25 chimera determined as described in Experimental Procedures and expressed in Miller units. NC, activity of bacteria producing T18 and T25 as negative control; PC, activity of positive control strain producing T18-Zip and T25-Zip chimera. Each bar represents mean betagalactosidase activity value from at least 3 independent colonies obtained by cotransformation of pKT25 and pUT18c derivatives encoding pseudopilin chimera (T25 or T18 fused to the N-terminus of mature pseudopilins) indicated by the colour code in the inset: PulG, red; PulH, purple; Pull, orange; PulJ, blue; and PulK, green. The colours of the bars correspond to the T25-fused proteins. The red horizontal line indicates the background mean beta-galactosidase activity measured in the negative control. Error bars indicate standard deviation. Statistical significance relative to the negative controls is indicated above graphs as follows: ${ }^{* * * *}=\mathrm{p}<0.001 ;{ }^{* * *}=\mathrm{P}<0.01 ;{ }^{*}=\mathrm{P}<0.1 ; \mathrm{ns}=$ non-significant. The inset above the main graph shows the part of the same graph with the scale of beta-galactosidase activities expanded in the low range (from 0 to 500 Miller units). B. The results are summarised schematically in a pentagram depicting pseudopilin arrangement in a putative right-handed helical complex. Arrows are oriented from T18- to T25- chimera and their line thickness indicates the strengths of significant interactions, in the range of beta-galactosidase activities (in Miller units) defined as shown in the legend: weak (50>mean $>500)$, strong $(500>$ mean $>1500)$ and very strong (mean $>1500)$. 
A

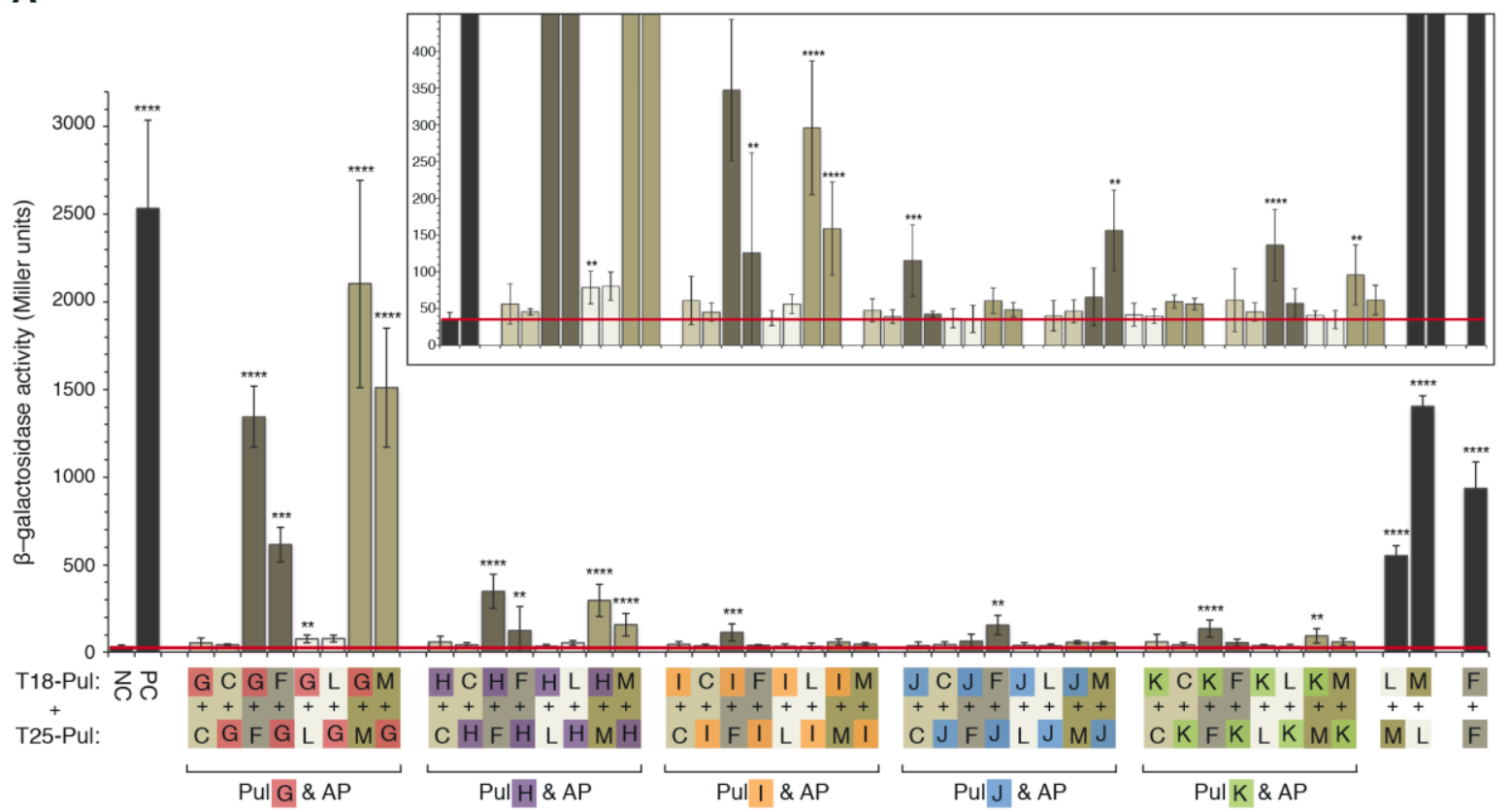

B

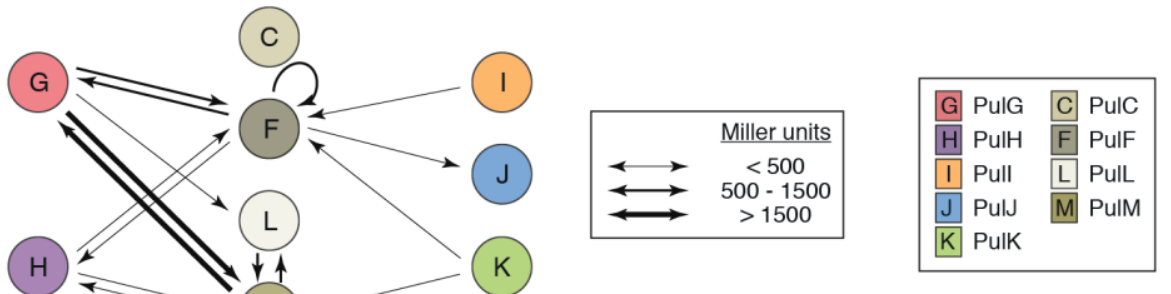

Figure 5. Interactions of pseudopilins with assembly platform components identified using bacterial two-hybrid analysis. A. Beta-galactosidase activities of DHT1 bacteria producing indicated T18-Pul or T25-Pul hybrids as bait in the presence of AP component chimera. The Pul components analysed are indicated by the single letter and colour code in the inset. NC, negative control strain co-producing T18 and T25 fragments; PC, positive control coproducing T18-Zip and T25-Zip chimera. The bars represent mean values from at least 3 independent colonies resulting from co-transformation of strain DHT1 with indicated pKT25 and pUT18c derivatives. The colours of the bars correspond to the AP proteins tested. The red line indicates the background beta-galactosidase activity measured in the NC. Error bars indicate standard deviation. Statistically significant mean values relative to the negative control (Experimental Procedures and Supplementary Dataset 1) are indicated above bars. The inset above the main graph shows the part of the same graph with the expanded scale of beta-galactosidase activities in the low range (from 0 to 500 Miller units). B. Summary of the interaction data, with the arrows oriented from T18- to T25- chimera and with line thickness corresponding to arbitrary range of interaction strengths indicated in the legend, as in Figure 4. 
A

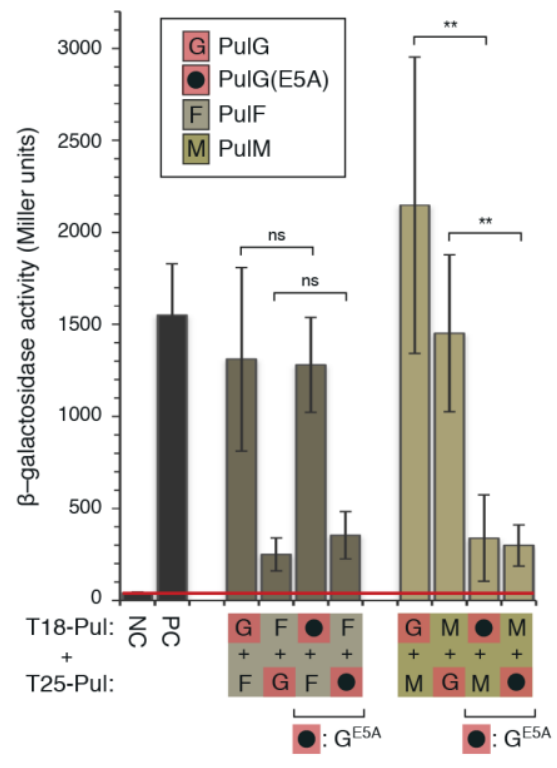

B

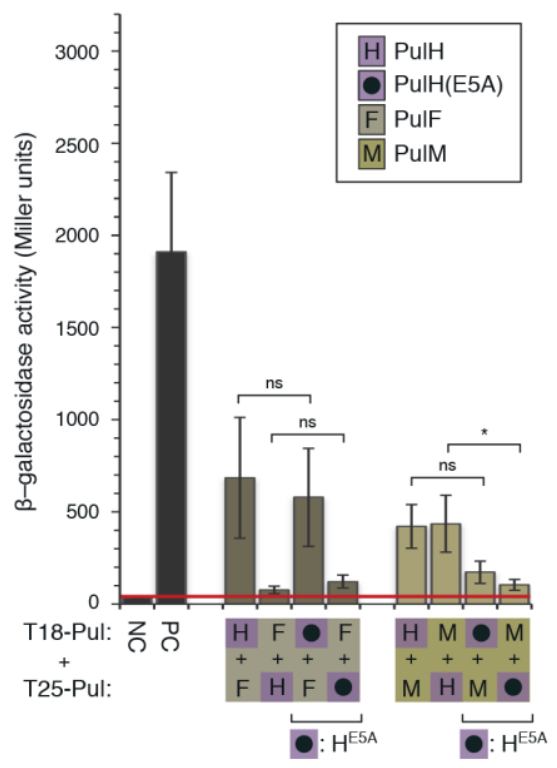

875

Figure 6. The effect of E5A substitution on PulG and PulH interactions with PulF and PulM. A. Bacterial two-hybrid analysis of PulG and PulG ${ }^{E 5 A}$ interactions with PulF and PulM. B. Bacterial two-hybrid analysis of $\mathrm{PulH}$ and $\mathrm{PulH}^{\mathrm{E} 5 \mathrm{~A}}$ interactions with PulF and PulM. Each bar represents the mean value from at least 3 independent colonies obtained by transformation of pKT25 and pUT18c derivatives containing indicated inserts. The colours of the bars correspond to the AP proteins. Error bars indicate standard deviation. NC, negative control and PC, positive control as indicated in the legend of Figure 4. The red line indicates the background beta-galactosidase activity of the NC. Statistical significance of multiple pairwise comparisons is indicated by stars, as in Figure 4. 


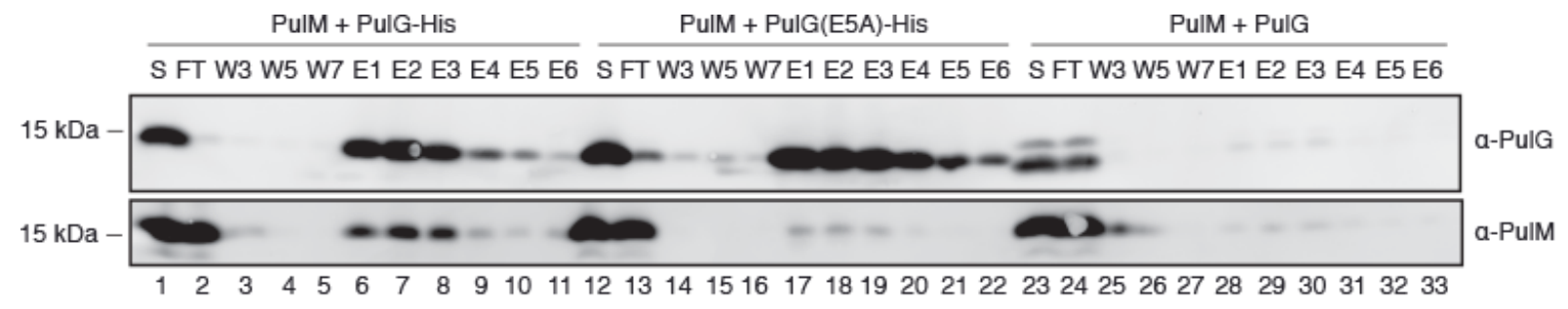

886

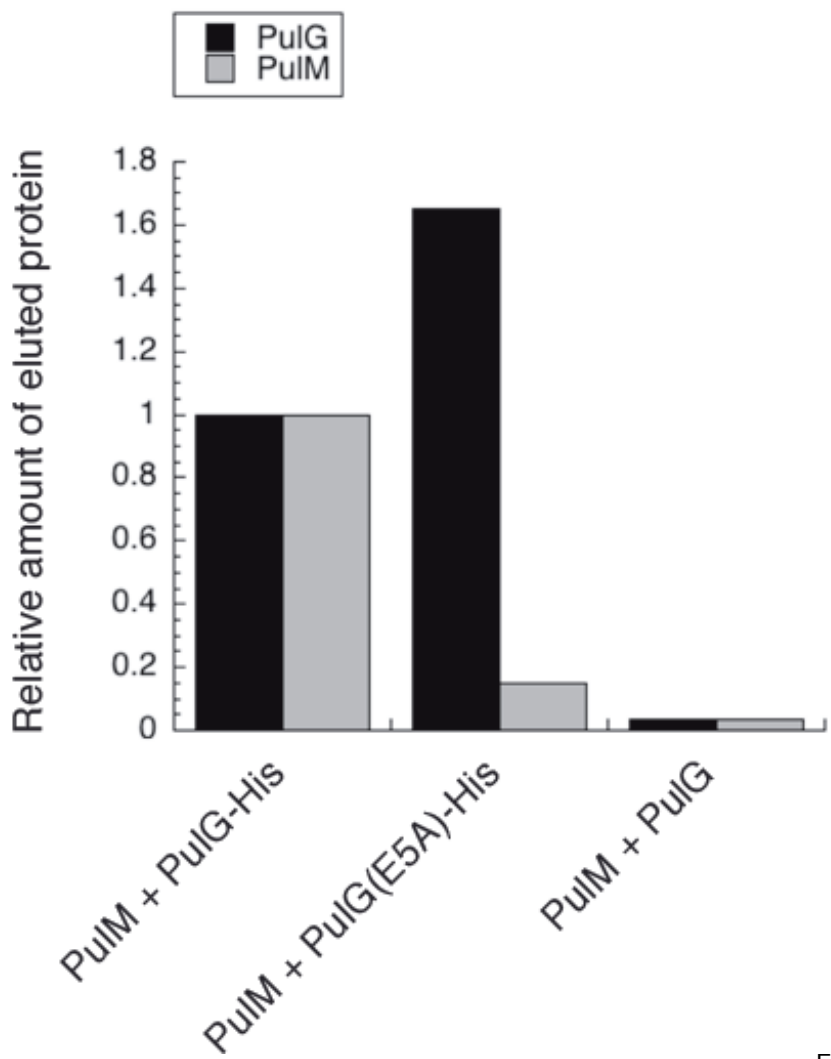

Figure 7. Co-purification of PulM with PulG-His ${ }_{6}, \mathrm{PulG}^{\mathrm{E}}{ }^{-}-\mathrm{His}_{6}$ and PulG. Triton X-100 soluble extracts of total membranes from E. coli strains PAP7460 producing PulM and the indicated PulG variants were subject to affinity chromatography on Ni-NTA matrix (Experimental Procedures). A. SDS-PAGE and immuno-detection of PulG and PulM in fractions of the co-purification. S, solubilised membrane fractions; FT, flow-through; W1, W3 and W7, wash fractions; E1-E6, elution fractions. B. Quantification of the relative amount of eluted PulG and PulM relative to wild type levels, according to the densitometric analysis of the bands in $(A)$. 
Assembly

(1)

(2)

(3)

(4)

(5)

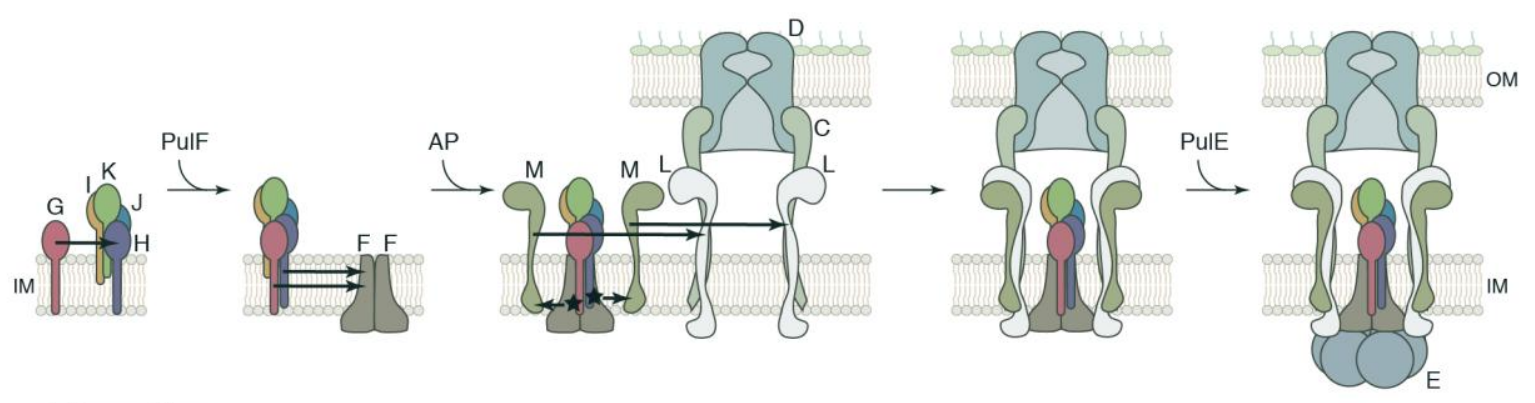

Elongation

(6)

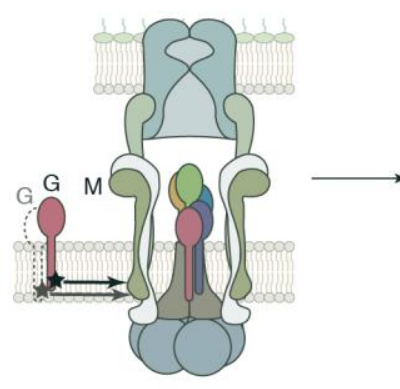

(7)

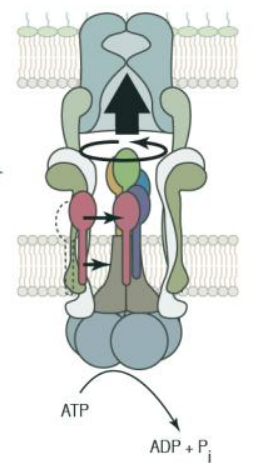

(8)

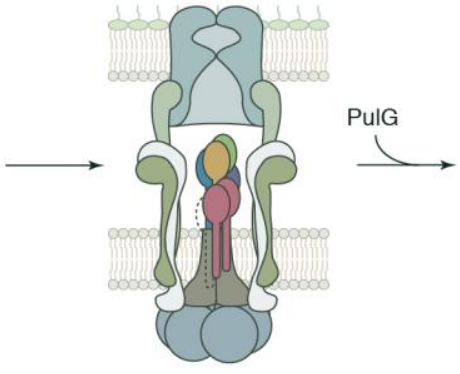

896

Figure 8. The working model of pseudopilus assembly and elongation. Assembly (top panel): Mature major pseudopilin PulG interacts with the quaternary complex composed of the priming PulJ-Pull-PulK trimer bound to PulH (step 1). Transmembrane regions of PulG and PulH drive the association of the pentameric proto-pseudopilus to a PulF dimer (2). PulM binds to this complex via the specific interactions of PulM with E5 residues of PulG and PulH, depicted as stars (3). The PulF-pseudopilin-PulM complex associates with PulL within the pre-assembled secretin PuID in the OM bound to the IM protein PulC. The initiation complex (4) recruits hexameric ATPase PulE via the cytoplasmic regions of PulL and possibly via PulF, thus resulting in a complete, functional T2S machine (5). Elongation (bottom panel): Fibre elongation begins with the recruitment of PulG subunits, likely in the form of dimers, by PulM (6). As in step 3, this recruitment requires a direct contact between the E5 residue of PulG and PulM (6). PulG enters the assembly platform, docks to PulF via its TM segments and associates with PulG ${ }^{\mathrm{P}+1}$ protomer of the proto-pseudopilus via their globular domains (7), through electrostatic contacts described previously (Nivaskumar et al., 2014). ATP hydrolysis causes conformational changes in PulE that are transmitted to PulF through direct contact, driving the rotation of the proto-fibre (7). The incoming PulG protomer is spooled into the fibre, which results in an overall extension of the pseudopilus (7 and 8). Fibre growth comprises multiple cycles of PulG recruitment, docking and extraction (steps 6 to 9). 